\title{
Lightweight and Static Verification of UML Executable Models
}

\author{
Elena Planas ${ }^{\mathrm{a}, *}$, Jordi Cabot ${ }^{\mathrm{b}, \mathrm{a}}$, Cristina Gómez $^{\mathrm{c}}$ \\ ${ }^{a}$ Universitat Oberta de Catalunya (Spain) \\ ${ }^{b}$ ICREA (Spain) \\ ${ }^{c}$ Universitat Politècnica de Catalunya (Spain)
}

\begin{abstract}
Executable models play a key role in many software development methods by facilitating the (semi)automatic implementation/execution of the software system under development. This is possible because executable models promote a complete and fine-grained specification of the system behaviour. In this context, where models are the basis of the whole development process, the quality of the models has a high impact on the final quality of software systems derived from them. Therefore, the existence of methods to verify the correctness of executable models is crucial. Otherwise, the quality of the executable models (and in turn the quality of the final system generated from them) will be compromised. In this paper a lightweight and static verification method to assess the correctness of executable models is proposed. This method allows to check whether the operations defined as part of the behavioural model are able to be executed without breaking the integrity of the structural model and returns a meaningful feedback that helps repairing the detected inconsistencies.
\end{abstract}

Keywords: Model-Driven Development (MDD), Model-Driven Architecture (MDA), Executable Models, Verification, Static Analysis, Alf Action Language

\section{Introduction}

Executable models are models with a behavioural specification detailed enough so that they can be systematically implemented or executed in the production environment. Executable models play a cornerstone role in the Model-Driven Development (MDD) paradigm, where models are the core artifacts of the development life-cycle and the basis to generate the final software implementation.

Executable models are not a new concept (e.g. [31, 58]) but are now experiencing a comeback, becoming a relevant topic within the OMG (Object

\footnotetext{
* Corresponding author at: Rambla del Poblenou 156, 08018 Barcelona, Spain. Tel.: +34 9332635 49; fax: +3493326 8822 .

Email addresses: eplanash@uoc.edu (Elena Planas), jordi.cabot@icrea.cat (Jordi Cabot), cristina@essi.upc.edu (Cristina Gómez)
}

Preprint submitted to Computer Languages, Systems and Structures

January 13, 2017 
Management Group). Their use is being promoted given the value they can bring. As one of its creators declares "executable models increase productivity by raising the level of abstraction; reduce costs by describing systems independently of their implementation; and improve the quality of the final system by facilitating early verification" [37].

Following this trend, the OMG has published in the last years several versions of the Foundational Subset for Executable UML Models (fUML) standard [46], an executable subset of the UML that can be used to define, in an operational style, the semantics of systems. The OMG has also published the first standard version of the Action Language for Foundational UML (Alf) standard [45], a textual surface notation which maps to the fUML [46], that provides the constructs to specify the fine-grained behaviour of systems in terms of actions. As can be seen, the increasing implementation of these standards in modeling tools $[19,39,11]$ shows a raising interest for this topic.

Given the growing importance of executable models and the impact of their correctness on the final quality of software systems derived from them [41], the existence of methods to verify the correctness of such models is becoming crucial. Otherwise, the quality of the executable models (and in turn the quality of the final system generated from them) will be compromised.

In this paper we propose a method (see Figure 1) focused on the verification of the executability of operations specified by means of actions (i.e. actionbased operations) with respect to a subset of the integrity constraints that can appear in a model. We consider this is an important criteria to preserve the correctness of such models. Besides checking the executability of the operations, the method we propose returns a meaningful feedback that helps repairing the detected inconsistencies.

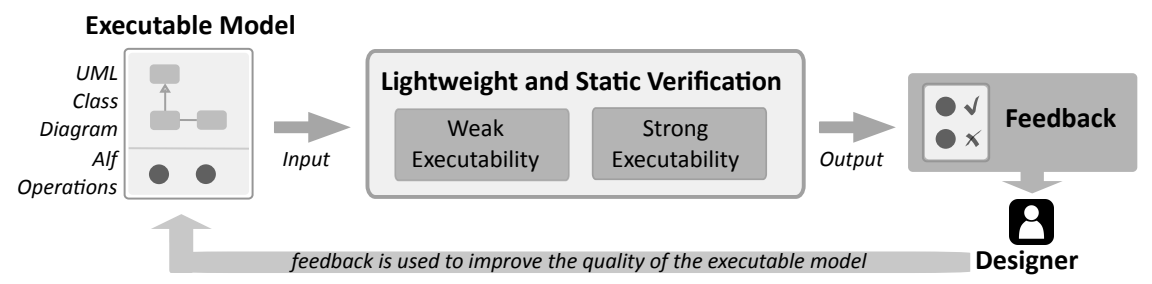

Figure 1: Method overview.

Like other studies that focus on executability $[12,13,55]$, our method classifies the operations in three categories:

1. Strongly executable (SE) operations, i.e. operations that are guaranteed to always generate a consistent state. We know for sure that all executions of the operation (regardless of the input values provided to the operation and the initial system state where the operation is applied on) reach a consistent state with respect to the structural model and their integrity constraints.

2. Weakly executable (WE) operations, i.e. operations that sometimes generate a consistent state, but are not guaranteed to do so. We can ensure 
that at least one of the many possible executions of the operation during the life span of the system will be successfully executed but probably not all of them (e.g. depending on the input parameters).

3. Non executable $(\neg \mathbf{E})$ operations, i.e. operations that never generate a consistent system state. After their execution, they always reach a state that violates some integrity constraints of the structural model (e.g. some cardinality constraints). Note that this does not mean that the operations cannot be executed, but that their execution always generate an inconsistent system state.

$\neg$ E operations, when executed as standalone operations, are useless since every time a user tries to execute them (regardless the provided input values) an error arises because some integrity constraints become violated. Also notice that weak executability is a necessary (but not sufficient) condition for strong executability. Then SE operations are a subset of WE operations.

In contrast with most related works, our method follows a lightweight approach. The term lightweight was popularized over almost twenty years ago following the publication of a round-table article [56], which invited the use of formal methods in a practical way. We consider our method as being lightweight since it performs a static analysis of the model, i.e. it examines the model without executing its operations [40]. Static analysis has been mainly applied to analyze the source code of programs [54], but the same idea may be extended to analyze models at design time without requiring any kind of simulation. Besides, our method provides valuable information as feedback (in case of error, it points out the source of the error and assists the designer during their correction). As we will see later, our method relies on some assumptions regarding the input model and requires some trade-offs to enable our lightweight analysis.

The work reported here extends our previous works [51] and [52] on several directions: (1) We increase the expressiveness of the models our method can deal with; (2) In order to align our work with the new UML standards, we now specify the operations by means of the Alf action language [45] provided by the OMG; (3) We re-design our previous methods [51, 52] to provide a unique and integrated method for verifying weak and strong executability properties; (4) In our previous work [52] we only provide the skeleton of the method as a black box, while in this work we elaborate in detail each step of the method; and (5) We provide a prototype tool that implements our method.

To the best of our knowledge, only few works have been devoted to verify fUML/Alf specifications [4, 8, 17, 33, 35, 38]. As suggested in [42, 24] and more specifically in [49], the verification of such specifications should be studied.

Paper Organization. The rest of the paper is structured as follows. Section 2 presents the motivation of our work. Section 3 introduces the basic concepts and the notation that will be used in the rest of the paper. Section 4 defines the concepts of execution paths and executability. Section 5 describes our method, Section 6 presents the prototype tool that implements it, Section 7 describes an empirical evaluation to assess the usefulness and the efficiency of our method, and Section 8 discusses about its pros and cons. Finally, Sec- 
tion 9 presents the related work and Section 10 summarizes with the relevant conclusions and further work.

\section{Motivation}

Executability is one of the most fundamental correctness properties for behavioural models. As we introduced, operations can be classified as strongly executable (SE), weakly executable (WE) or non executable $(\neg \mathrm{E})$.

The previous properties can also be characterized in terms of probabilities: $\neg \mathrm{E}$ operations generate a consistent state with probability 0 , WE operations generate a consistent state with probability strictly greater than 0 and SE operations generate a consistent state with probability exactly 1 . Then, WE operations may be viewed as optimistic operations since they apply the changes to the system state without ensuring that those changes will not break any constraint. They hope that they will not but an external mechanism is needed to check this and act accordingly (e.g. rolling back the changes if they indeed violate the constraints). Instead, SE operations may be viewed as safe operations (they will be successfully executed in any scenario).

Generally speaking ${ }^{1}$, making sure that all operations are WE ensures a basic level of correctness. However, there might be cases (for instance, some critical and/or concurrent systems) in which WE operations are not sufficient. In these cases we believe it is also necessary to guarantee SE operations. Making sure that all operations are SE at design time ensures a hight level of correctness, by facilitating a lot the development (and run-time efficiency) of the system: since we know that operations always leave the system in a consistent state, we can avoid checking at the end of each operation execution whether all constraints are satisfied which improves the efficiency of the system. Building (and executing) such integrity checking mechanism is an error-prone and time-consuming process that can be avoided when using our method.

Note that our purpose is to verify operations (ensuring operations are correct) and not to validate them (ensure they are the correct operations to implement the user expectations).

Executability is not a new property, it has been studied, for instance, to verify declarative operations $[13,55]$ and model-to-model transformations [12, 53] (for more details, see the related work in Section 9). However, there is a lack of methods to check this property on executable models.

\subsection{The Need for Verification: Writing Executable Operations Is Not Easy}

In general, we cannot assume that designers are able to easily write executable operations by themselves. To prove this fact, we did an experiment

\footnotetext{
${ }^{1}$ Strictly speaking, and as we will discuss in Section 5 , this only needs to apply to those operations that can be directly executed by the user as a kind of single transaction (i.e. an operation that guarantees an atomic and isolated execution, a consistent system state after its execution and the durability of execution results [28]). Then, helpers and other operations that will not be executed as standalone operations may be discarded from our analysis.
} 
with a total of one hundred students of a Software Engineering course of the Computer Science Degree taught at the Open University of Catalonia (UOC), all of them had a good background about modeling and programming. The students were provided with a UML class diagram (containing 14 classes, 10 associations and 32 integrity constraints) representing a car sharing system and they were asked to design four action-based operations. All operations were of low and medium complexity in terms of the number of actions they contained (an average of 6.5 actions each operation).

The results (see Figure 2) showed that, among all the operations designed by the students, $67 \%$ of them were $\neg \mathrm{E}, 33 \%$ of them were WE and only $26 \%$ were also SE. Figure 3 shows the frequency of the typical errors found in the operations designed by the students.

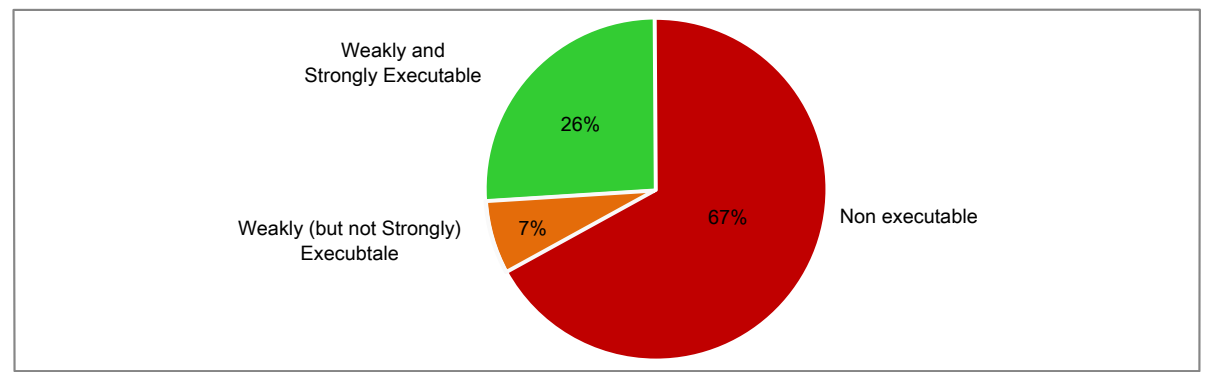

Figure 2: Results of our experiment.

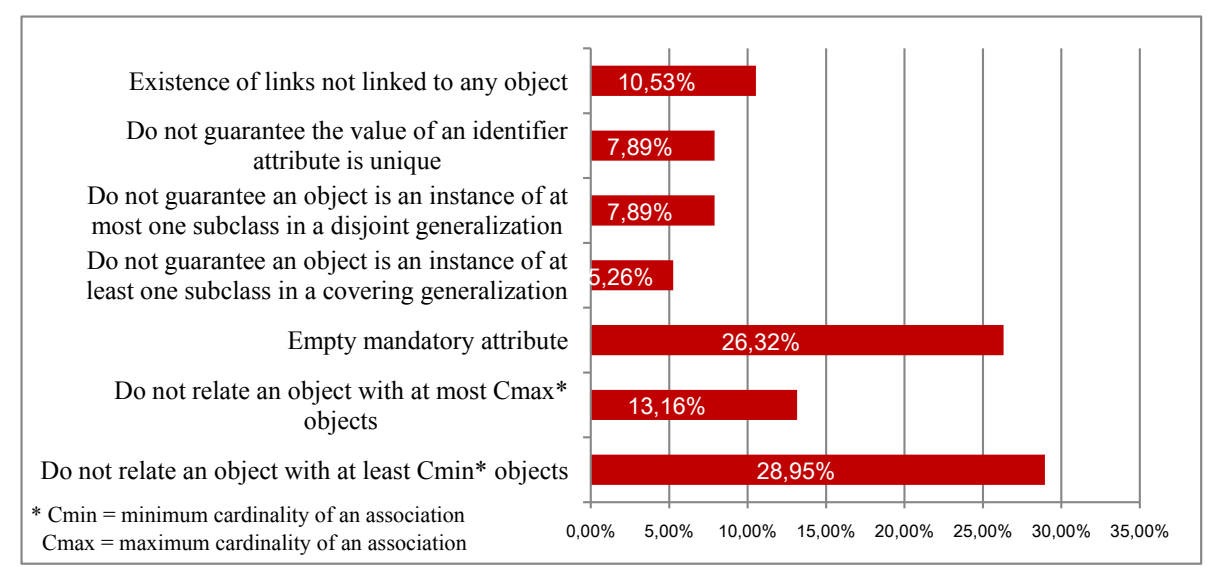

Figure 3: Frequency of the errors found in the operations of our experiment.

We believe this experiment supports the need for a method able to evaluate the correctness of the operations and that can be easily integrated in the modeling tools used by practitioners. 


\section{Basic Concepts and Notation}

In order to facilitate the comprehension of our method, this section describes the basic concepts that will be used in the rest of the paper. In the following, the concepts of executable model (Section 3.1), structural model (Section 3.2) and behavioural model (Section 3.3) are reviewed.

In this paper we assume that executable models are written using the OMG standards (UML [47], OCL [44] and Alf [45] languages). Our choice is based on the wide adoption of the OMG standards in modeling specifications, although many concepts of this work are applicable to other languages as well.

\subsection{Executable Model}

It is widely accepted that a model is a simplified representation of a complex reality [10]. Moreover, an executable model is a model with a behavioural specification detailed enough so that it can be systematically implemented or executed in the production environment.

We represent an executable model $(E x M)$ as a 2-tuple $\langle S M, B M\rangle$, where $S M$ is a structural model and $B M$ is a behavioural model.

\subsection{Structural Model}

A structural model specifies the static part of a software system, i.e. the general knowledge about the system domain [43].

We represent an structural model $(S M)$ as a UML class diagram, composed by a set of classes, a set of attributes of each class, a set of binary associations and generalizations among classes and a set of integrity constraints (i.e. conditions that must be satisfied in all states of a software system [43]). Some integrity constraints (for instance, cardinalities and disjointness/covering constraints in generalizations) may be graphically represented in the class diagram, while others can be textually specified in OCL [44].

When verifying the executability of operations, our method takes into account the most commonly used integrity constraints according to [16]. They are shown in Table 1. First column (Contraint) identifies the constraint type, second column (Abbreviation) indicates the precise notation that our method internally uses, third column (Description) describes the meaning of the constraint, and last column (Formalization in $O C L$ ) provides its formal description. Some new constraints could be added to this table, however, as we discuss in Section 8 our method is not suitable to address arbitrary integrity constraints.

Table 1: Constraint types supported by our method.

\begin{tabular}{|c|c|c|c|}
\hline Constraint & Abbreviation & Description & Formalization in $O C L$ \\
\hline $\begin{array}{l}\text { Minimum } \\
\text { cardinality } \\
\text { of a class }\end{array}$ & $\operatorname{Cmin}(\mathrm{cl})$ & $\begin{array}{l}\text { Expresses the mini- } \\
\text { mum objects of class } \\
\text { cl that must exist } \\
\text { simultaneously }\end{array}$ & $\begin{array}{l}\text { context cl inv: } \\
\text { cl.allinstances () ->size() } \\
>=\text { Cmin (cl) }\end{array}$ \\
\hline $\begin{array}{l}\text { Maximum } \\
\text { cardinality } \\
\text { of a class }\end{array}$ & $\operatorname{Cmax}(\mathrm{cl})$ & $\begin{array}{l}\text { Expresses the maximum } \\
\text { objects of class } \mathrm{cl} \text { that } \\
\text { may exist simultaneously }\end{array}$ & $\begin{array}{l}\text { context cl inv: } \\
\text { cl.allinstances () ->size() } \\
<=\operatorname{Cmax}(\mathrm{cl})\end{array}$ \\
\hline
\end{tabular}


Table 1 - continued from previous page

\begin{tabular}{|c|c|c|c|}
\hline Constraint & Abbreviation & Description & Formalization in $O C L$ \\
\hline $\begin{array}{l}\text { Minimum } \\
\text { cardinal- } \\
\text { ity of an } \\
\text { association }\end{array}$ & $\operatorname{Cmin}(a, r, r)$ & $\begin{array}{l}\text { Expresses the minimum } \\
\text { multiplicity of the mem- } \\
\text { ber end (i.e. role) r } \\
\text { of an association as be- } \\
\text { tween cl (with role r) } \\
\text { and } \mathrm{cl}^{\prime}\end{array}$ & $\begin{array}{l}\text { context cl inv: } \\
\text { cl.r->size }() \quad>=\operatorname{Cmin}(\text { as }, r)\end{array}$ \\
\hline $\begin{array}{l}\text { Maximum } \\
\text { cardinal- } \\
\text { ity of an } \\
\text { association }{ }^{2}\end{array}$ & $\operatorname{Cmax}($ as,r) & $\begin{array}{l}\text { Expresses the maximum } \\
\text { multiplicity of the mem- } \\
\text { ber end (i.e. role) r } \\
\text { of an association as be- } \\
\text { tween cl (with role r) } \\
\text { and cl' }\end{array}$ & $\begin{array}{l}\text { context cl inv: } \\
\text { cl.r }()->\operatorname{size}()<=\operatorname{Cmax}(\text { as, }, r)\end{array}$ \\
\hline $\begin{array}{l}\text { Mandatory } \\
\text { attribute }\end{array}$ & $\begin{array}{l}\text { Mand(attr, } \\
\text { cl) }\end{array}$ & $\begin{array}{l}\text { Expresses the attribute } \\
\text { attr of class } c l \text { must } \\
\text { have at least one value }\end{array}$ & $\begin{array}{l}\text { context cl inv: not } \\
\text { cl.attr->oclisundefined() }\end{array}$ \\
\hline $\begin{array}{l}\text { Covering of } \\
\text { a generaliza- } \\
\text { tion }\end{array}$ & $\begin{array}{l}\operatorname{Cov}(\mathrm{cl}, \\
\left\{c l_{1}, \ldots,\right. \\
\left.\left.c l_{n}\right\}\right) \quad(\mathrm{cl} \\
\text { generalizes } \\
\left.c l_{1}, \ldots, c l_{n}\right)\end{array}$ & $\begin{array}{l}\text { Requires each instance of } \\
\text { cl to be an instance of at } \\
\text { least one } c l_{i} \text {. }\end{array}$ & $\begin{array}{l}\text { context cl inv: } \\
\text { self.oclistypeof }\left(c l_{1}\right) \text { or } \\
\ldots \text { or self.oclistypeof }\left(c l_{n}\right)\end{array}$ \\
\hline $\begin{array}{l}\text { Disjointness } \\
\text { of a general- } \\
\text { ization }\end{array}$ & $\begin{array}{l}\operatorname{Disj}(c l, \\
\left\{c l_{1}, \ldots,\right. \\
\left.\left.c l_{n}\right\}\right) \quad(c 1 \\
\text { generalizes } \\
\left.c l_{1}, \ldots, c l_{n}\right)\end{array}$ & $\begin{array}{l}\text { Requires each instance of } \\
\text { cl to be instance of at } \\
\text { most one } c l_{i}\end{array}$ & $\begin{array}{l}\text { context cl inv: } \\
\text { self.oclisTypeOf }\left(c l_{i}\right) \text { implies } \\
\text { not self.oclisTypeOf }\left(c l_{x}\right) \\
\text { where } x, i=1 . . n \text { and } x \neq i\end{array}$ \\
\hline Identifier $^{3}$ & $\mathbf{I D}($ attr, $\mathrm{cl})$ & $\begin{array}{l}\text { Expresses the attribute } \\
\text { attr uniquely identifies } \\
\text { instances of } \mathrm{cl}\end{array}$ & $\begin{array}{l}\text { context cl inv: } \\
\text { cl.allInstances () }-> \\
\text { isunique (attr) }\end{array}$ \\
\hline $\begin{array}{l}\text { Symmetry of } \\
\text { a recursive } \\
\text { association }\end{array}$ & $\operatorname{Sym(as)}$ & $\begin{array}{l}\text { Guarantees if an object } \\
o_{1} \text { is as-related to } o_{2}, \\
\text { then } o_{2} \text { is as-related to } \\
o_{1}\end{array}$ & $\begin{array}{l}\text { context cl inv: self.r } \\
->\text { forAll(o|o.r }-> \\
\text { includes (self)), where } r \text { is } \\
\text { a member end of as }\end{array}$ \\
\hline $\begin{array}{l}\text { Asymmetry } \\
\text { of a re- } \\
\text { cursive } \\
\text { association }\end{array}$ & $\operatorname{Asym(as)}$ & $\begin{array}{l}\text { Guarantees that if an ob- } \\
\text { ject } o_{1} \text { is as-related to } \\
o_{2} \text {, then } o_{2} \text { is not as- } \\
\text { related to } o_{1}\end{array}$ & $\begin{array}{l}\text { context cl inv: self.r } \\
->\text { forAll (o|o.r }-> \\
\text { excludes (self)), where } r \text { is } \\
\text { a member end of as }\end{array}$ \\
\hline $\begin{array}{l}\text { Irreflexivity } \\
\text { of a re- } \\
\text { cursive } \\
\text { association }\end{array}$ & Irrefl(as) & $\begin{array}{l}\text { Guarantees that an ob- } \\
\text { ject } o \text { is never as-related } \\
\text { to itself }\end{array}$ & $\begin{array}{l}\text { context cl inv: } \\
\text { self.r->excludes (self), where } \\
r \text { is a member end of as }\end{array}$ \\
\hline $\begin{array}{l}\text { Value com- } \\
\text { parison }\end{array}$ & $\begin{array}{l}\text { ValueComp } \\
(\text { attr, op, v) }\end{array}$ & $\begin{array}{l}\text { States a restriction on } \\
\text { the value of the at- } \\
\text { tribute attr: the expres- } \\
\text { sion attr op } \mathrm{v} \text { (where } \\
\text { op is a comparison op- } \\
\text { erator and } \mathrm{v} \text { is a value) } \\
\text { must be true }\end{array}$ & $\begin{array}{l}\text { context cl inv: self.attr } \\
\langle o p>V\end{array}$ \\
\hline $\begin{array}{l}\text { Referential } \\
\text { integrity } \\
\text { constraint }\end{array}$ & $\begin{array}{l}\text { Referential } \\
(c 1, \text { as })\end{array}$ & $\begin{array}{l}\text { Guarantees each partic- } \\
\text { ipant in the association } \\
\text { as (in which cl partic- } \\
\text { ipates) is an instance of } \\
\text { its corresponding class }\end{array}$ & $\begin{array}{l}\text { context cl inv: not } \\
\text { self.r->oclisundefined(), } \\
\text { where } r \text { is a member end of as }\end{array}$ \\
\hline
\end{tabular}

The state of a system at a specific time is the set of instances of the classes and associations defined in the class diagram that exist at that time [43]. It can be represented in UML using an object diagram.

\footnotetext{
${ }^{2}$ Multivalued attributes are treated as associations, since they behave in the same way.

${ }^{3}$ Although the latest version of UML [47] allows to represent the constraint ID, it does not define any associated notation. This is the reason why we represent this constraint by OCL.
} 


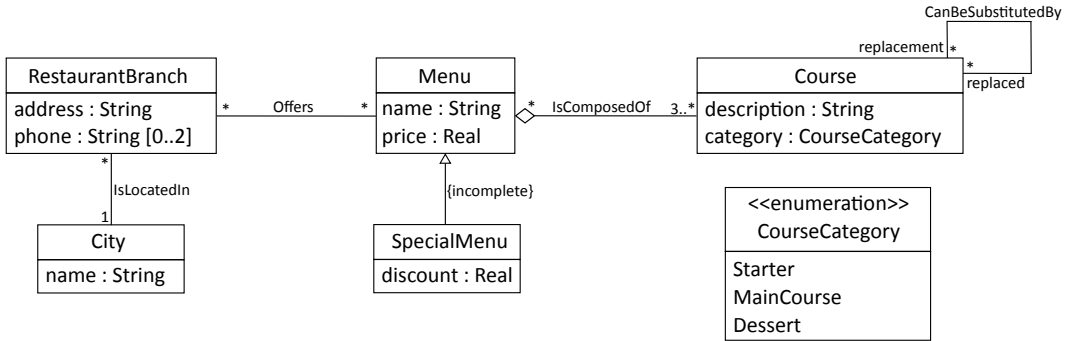

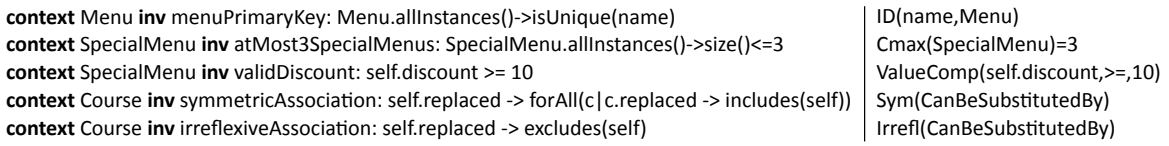

Figure 4: Excerpt of a restaurant chain class diagram.

Example 1 Given the class diagram of Figure 4, a possible system state called currentState would be a state in which there is a restaurant branch with address "Camèlies Street, 53, Barcelona", which offers a non-special menu called "Anticrisis menu" for $5 €$ (see Figure 5) .

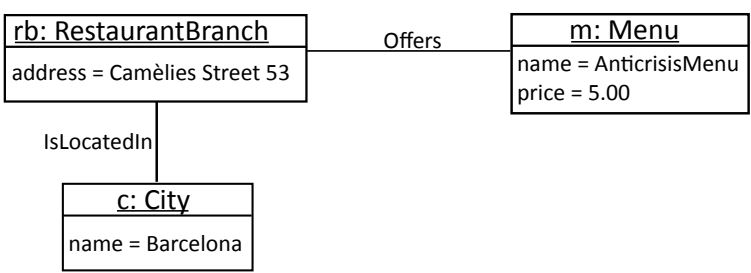

Figure 5: Object diagram representing the state currentState described in Example 2.

A state $s$ satisfies an integrity constraint $i c$ iff $i c$ evaluates to true in this state. We denote by Satisfies $(s, i c)$ the proposition that states that $s$ satisfies the integrity constraint $i c$. Otherwise, we say that the constraint is unsatisfied or violated.

Let $E x M=\langle S M, B M\rangle$ be an executable model, a system state $s$ is consistent regarding $S M$ iff $\forall i c \in S M$, Satisfies(s,ic). We denote by IsConsistent $(s, S M)$ the proposition that states that $s$ is consistent regarding the structural model $S M$.

Example 2 The state currentState (see Figure 5) does not satisfy the minimum cardinality constraint of the association Iscomposedof in the role course, since the menu "Anticrisis menu" does not contain any course, i.e. Satisfies(currentState, Cmin (IsComposedof, course)=3) $=$ false. 
Therefore, this state is not consistent with the structural model of Figure 4, i.e. IsConsistent(currentState, RestaurantChain_CD) = false.

\subsection{Behavioural Model}

A behavioural model specifies the dynamic part of a software system, i.e. the valid changes in the system state, as well as the functions that the system can perform [43]. In UML there are several alternatives to represent the behaviour of a system but low-level specifications typically rely on the specification of operations (attached to UML classes) that are sequences of atomic steps that the users may execute to query/modify the information of the structural model. In this paper, we assume that a behavioural model (BM) is composed of a set of operations $\left\langle o p_{1}, \ldots, o p_{n}\right\rangle$, where each $o p_{i}$ is a sequence of Alf actions [45].

Alf is a standard published at the end of 2013 by the OMG (first beta version appeared in 2010). It provides a concrete syntax in charge of defining the basic read/write actions that can be used to specify the fine-grained behavioural aspects of systems plus some control flow statements to coordinate these actions in action sequences, conditional blocks or loops. The expressiveness of Alf is comparable to that of the instructions in traditional programming languages but at a higher abstraction (and platform-independent) level.

Table 2 shows the main modification actions provided by fUML (1st column), the corresponding concrete syntax provided by Alf (2nd column) and the description of the update they perform (3rd column). fUML predefines additional actions not shown in Table 2 since they do not affect our analysis.

Table 2: Main modification actions provided by fUML and concrete syntax in Alf.

\begin{tabular}{|c|c|c|}
\hline fUML Action & Alf Syntax & Description \\
\hline CreateObject & $\langle$ object $\rangle=$ new $<$ class $>()$ & $\begin{array}{l}\text { Creates and returns a new object of } \\
\text { type class }\end{array}$ \\
\hline DestroyObject & <object $>$.destroy () & $\begin{array}{l}\text { Destroys the object object from its } \\
\text { class and from any immediate super- } \\
\text { classes (if such exists) }\end{array}$ \\
\hline ReclassifyObject & $\begin{array}{l}\text { classify <object }>\text { [from } \\
<0 l d C l>][\text { to }<\text { newCl }>]\end{array}$ & $\begin{array}{l}\text { Removes object from classes in oldCl } \\
\text { and/or adds it as a new instance of } \\
\text { classes in newCl }\end{array}$ \\
\hline AddStructuralFeature & $\begin{array}{l}\begin{array}{l}\langle\text { object }>\cdot\langle\text { attribute }> \\
\langle\text { value }>\end{array} \\
\end{array}$ & $\begin{array}{l}\text { Sets value as the new value for the at- } \\
\text { tribute attribute of object object }\end{array}$ \\
\hline ClearStructuralFeature & $\begin{array}{l}\langle\text { object }>\text {.<attribute }>= \\
\text { null }\end{array}$ & $\begin{array}{l}\text { Removes all values for the attribute } \\
\text { attribute of object object }\end{array}$ \\
\hline CreateLink & $\begin{array}{l}<\text { association }>\text {.createLink } \\
(<\text { object } 1>,<\text { object } 2>)\end{array}$ & $\begin{array}{l}\text { Creates a new link }{ }^{4} \text { (i.e. associa- } \\
\text { tion instance) in the binary associa- } \\
\text { tion association between object } 1 \text { and } \\
\text { object2 }\end{array}$ \\
\hline DestroyLink & $\begin{array}{l}\text { <association>.destroyLink } \\
(<\text { object } 1>,<\text { object } 2>)\end{array}$ & $\begin{array}{l}\text { Destroys the link (i.e. } \\
\text { instance) in the binary association } \\
\text { association between object1 and } \\
\text { object2 }\end{array}$ \\
\hline ClearAssociation & $\begin{array}{l}\text { <association>.clearAssoc } \\
(<\text { object })\end{array}$ & $\begin{array}{l}\text { Destroys all links of the named } \\
\text { association that have at least one } \\
\text { end with value object }\end{array}$ \\
\hline OperationCall & $\begin{array}{l}{[<\text { result }>]=<\text { object }>\text {. }} \\
<\text { operation }>([\text { arguments }>])\end{array}$ & $\begin{array}{l}\text { Invokes the operation in the context } \\
\text { of the object with arguments and, op- } \\
\text { tionally, returns the result }\end{array}$ \\
\hline
\end{tabular}


Example 3 We show three operations (specified as UML activities in Alf) defined with Alf: (1) newCourse (in the context of class Course), creates a new course in the system; (2) addMenu (in the context of class Menu), adds a new menu to the system; and (3) classifyAsSpecialmenu (in the context of class Menu), classifies a menu as special menu. We consider all these three operations can be executed independently, then, they should be executable.

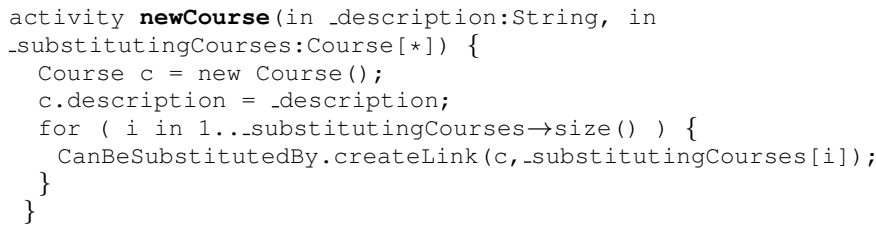

\section{Execution Paths and Executability}

The aim of this section is to precisely define the notion of executability regarding our two levels of correctness (weak and strong). With this purpose we need to first introduce the concept of execution path.

\subsection{Execution Paths}

The verification of the executability property is based on an analysis of the possible execution paths allowed by the actions that define the operation effect, i.e., the possible sequences of actions that may be followed during its execution.

In order to determine the execution paths, we propose to draw each operation as a Model-Based Control Flow Graph (MBCFG), a directed graph based on the model specification (instead of on the program code, as traditional control flow graph proposals). MBCFGs have also been used to express UML Sequence

${ }^{4}$ We assume that for every pair of objects there is at most one link in as between them. 
Diagrams [23]. We adapt this idea to express the control flow of action-based operations.

In order to represent Alf operations as MBCFGs, we consider the behaviour of each operation is an instance of exactly one Activity metaclass from fUML (see the fUML metamodel excerpt in Figure 6). We consider that each operation (i.e. Activity) consists in a sequence of actions ${ }^{5}$ (Action metaclass specializes the metaclass ExecutableNode which in turn specializes the metaclass ActivityNode). The list of available actions are all the subclasses of the Action metaclass as shown in Figure 6. This includes modification actions (also described in Table 2), ConditionalNodes (which represents an exclusive choice among a number of alternatives) and LoopNodes (that enables the definition of loops by means of a setup, test and body sections).

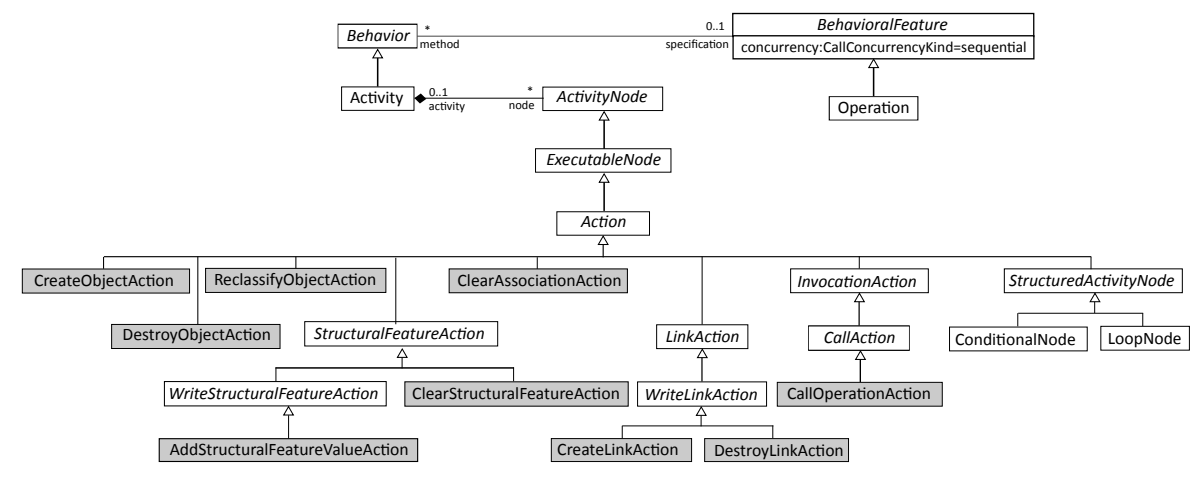

Figure 6: Fragment of the fUML metamodel supported by our method.

We also internally use two artificial control nodes that do not explicitly appear in Alf operations: the first node (representing the first instruction in the operation) and the last node (representing the last one). These two nodes help in clarifying the representation of our MBCFG.

Note that, since our method is focused on the correctness of single actionbased operations and not on the overall problem of verifying activity diagrams, it only supports a subset of the constructs that can appear on operations (those executable nodes included in Figure 6).

A Model-Based Control Flow Graph (MBCFG) for an operation op is a 2tuple $\left(V_{o p}, A_{o p}\right)$. The corresponding vertices $\left(V_{o p}\right)$ and arcs $\left(A_{o p}\right)$ are obtained applying the following rules:

- Every activity node (i.e. action) in $o p$ is a vertex in $V_{o p}$. In order to simplify the MBCFG, we only consider actions that may modify the system state (i.e. modification actions) and structured actions (i.e. conditionals or loops). It means that we skip other types of actions (as actions to read

\footnotetext{
${ }^{5}$ We ignore other types of ActivityNodes such as ControlNodes that can typically appear on activity diagrams but more rarely on operation specfications.
} 
values, to declare and initialize variables, and test actions to define the condition expression of a conditional/loop structure) since they do not affect the result of our analysis.

- An arc from a vertex $v_{1}$ to $v_{2}$ is created in $A_{o p}$ if $v_{1}$ immediately precedes $v_{2}$ in an ordered sequence of nodes.

- A vertex $v$ representing a conditional node $n$ is linked by an arc to the vertices $v_{1}, \ldots, v_{n}$ representing the first activity node for each clause (i.e. the then clause, the else clause, ...) in $n$. All vertices of each clause are englobed into a dashed line box. The last vertex in each clause is linked to the vertex $v_{\text {next }}$ immediately following $n$ in the sequence of executable activities. If $n$ does not include an else clause, an arc between $v$ and $v_{\text {next }}$ is also added to $A_{o p}$.

- Each arc from a conditional node to its first clause vertex is labelled with the condition of the conditional structure. Each arc to an else clause (or the arc between the conditional node and the $v_{n e x t}$ if there is not an else clause) is labelled with the negation of the above condition.

- A vertex $v$ representing a loop node $n$, is linked by an arc to the vertex representing the first activity node for n.bodyPart (returning the list of actions in the body of the loop) and the vertex $v_{\text {next }}$ immediately following $n$ in the activity. The last vertex in n.bodyPart is linked back to $v$ (to represent the loop behaviour).

- Each arc from a loop node to its first vertex is labelled with the condition fulfilled in the first execution of the loop followed by the times the loop is executed.

- A vertex representing an OperationCall action is replaced by the subdigraph corresponding to the called operation $o p^{\prime}$ as follows: (1) the initial vertex of $o p^{\prime}$ is connected with the vertex that precedes the OperationCall activity node in the main operation; (2) the final vertex of $o p^{\prime}$ is connected with the vertex/ces that follow the OperationCall; and (3) the parameters of $o p^{\prime}$ are replaced by the arguments in the call.

Example 4 Figures 7, 8 and 9 show the MBCFGs for the operations newCourse, addMenu and classifyAsSpecialMenu respectively, where each node has been labelled with a number.

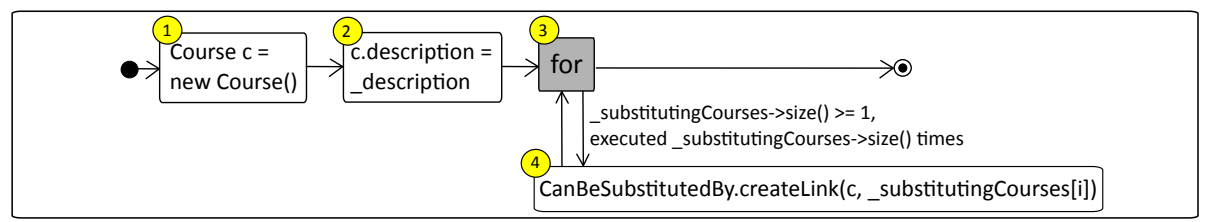

Figure 7: MBCFG of newCourse operation. 


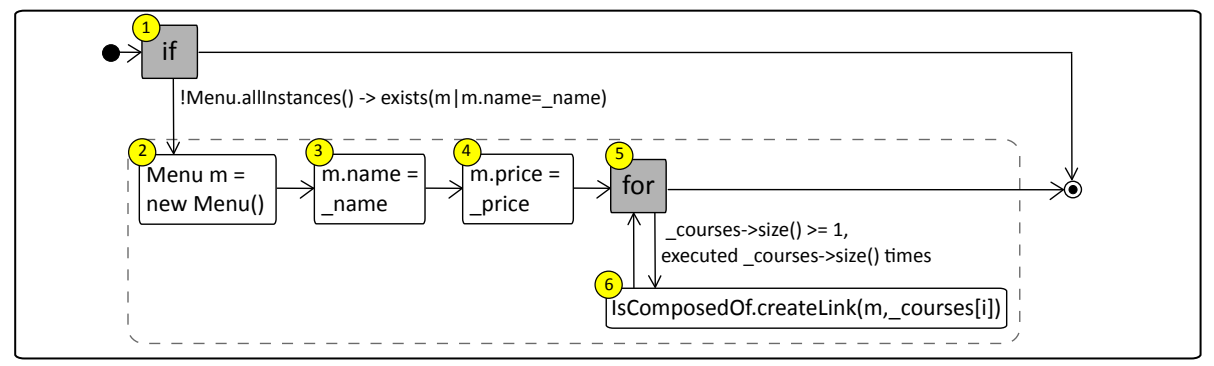

Figure 8: MBCFG of addMenu operation.

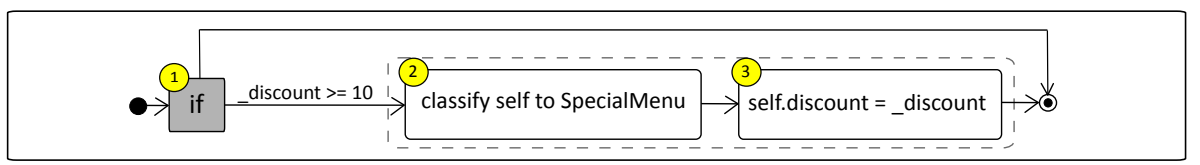

Figure 9: MBCFG of classifyAsSpecialMenu operation.

The process to verify the executability is based on an analysis of the possible execution paths allowed by the MBCFG. An execution path of an operation op is a finite and not empty sequence of actions that may be followed during the operation execution (note that empty paths are discarded). For trivial operations (e.g. with neither conditional nor loop nodes) there is a single execution path but, in general, several ones will exist.

Given a $M B C F G_{o p}$ for an operation op, the set of execution paths (Paths $(o p)$ ) for $o p$ is defined as Paths $(o p)=\operatorname{allPaths}\left(M B C F G_{o p}\right)$, where allPaths $\left(M B C F G_{o p}\right)$ returns the set of all paths in $M B C F G_{o p}$ that start at the initial vertex (the vertex corresponding to the initial node), end at the final vertex and do not include repeated arcs.

Example 5 Taking into account that empty paths are discarded, operations newCourse and addMenu have two execution paths (see Figures 10 and 11 respectively) and operation classifyAsSpecialMenu has a single execution path (see Figure 12).

\subsection{Executability}

The execution of an execution path $p$ over a system state $s$, generates a new state $s^{\prime}$ where the changes described in $p$ have been applied to $s$. We denote by AllExecutions $(p, s)=\left\{s_{1}^{\prime}, \ldots, s_{n}^{\prime}\right\}$ all the possible (potentially infinite) executions of the execution path $p$ over a system state $s$, that is, all the possible states $\left(s_{1}^{\prime}, \ldots, s_{n}^{\prime}\right)$ that may be reached (depending on the input arguments used to call the operation) by executing $p$ in $s$. 


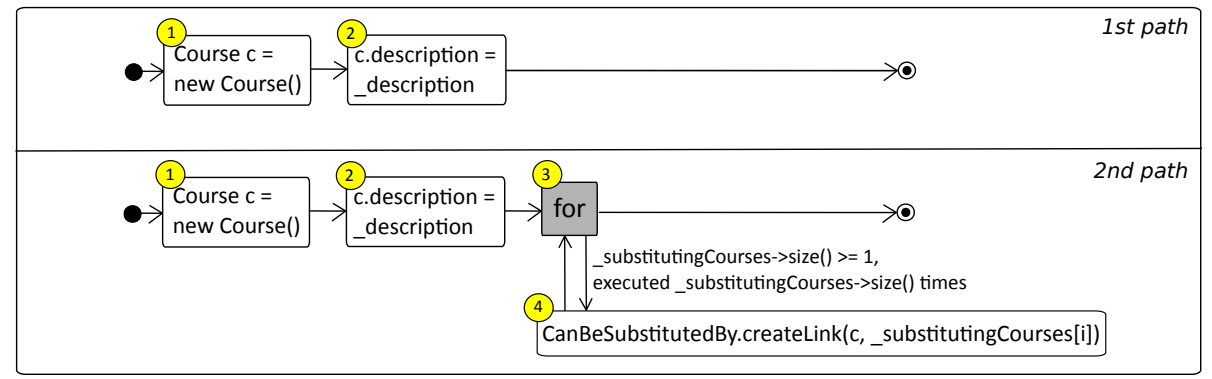

Figure 10: Paths of newCourse operation.

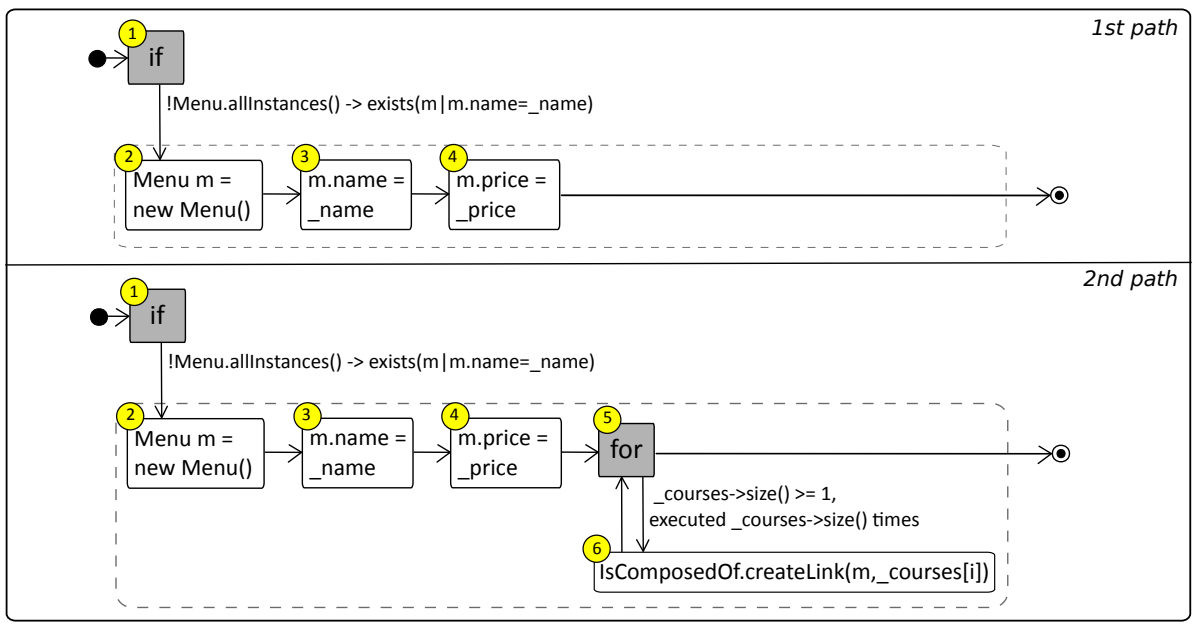

Figure 11: Paths paths of addMenu operation.

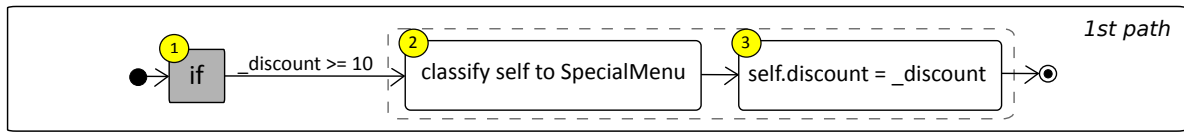

Figure 12: Unique path of classifyAsSpecialMenu operation.

\subsubsection{Weakly Executable Operations}

We consider an operation is weakly executable (WE) if it may generate a consistent state, but it is not guaranteed to do so. That is to say, an operation is WE if there is a chance that a user may successfully execute it, i.e. if there exists at least an initial state and a set of arguments for the operation parameters for which the execution of the actions included in the operation evolves the initial state of the system to a new state that satisfies all the integrity constraints of the structural model. Note that weak executability does not require the success of all executions of the operation to be successful. 
More formally:

Let $\mathrm{ExM}=\langle\mathrm{SM}, \mathrm{BM}\rangle$ be an executable model, an operation op $\in \mathrm{BM}$ is weakly executable (WE) iff $\exists \mathrm{p} \in \operatorname{Paths}(\mathrm{op}) \wedge \exists \mathrm{s}$ IsConsistent(s,SM) $\wedge \exists \mathrm{s}$ ' $\in$ AllExecutions $(\mathrm{p}, \mathrm{s}) \mid$ IsConsistent( $\left(\mathrm{s}^{\prime}, \mathrm{SM}\right)$

Example 6 Operation newCourse is not WE since it never may generate a consistent system state regarding the structural model of Figure 4: every time we try to create a new course $c$ but we do not assign any category for it, we reach an inconsistent system state where $c$ has no category, a situation forbidden by the structural model that defines the attribute category as mandatory (Mand(category,Course)), i.e. it must have at least one value. Instead, operation classifyAsSpecialMenu is WE since we are able to find an execution scenario (a system state that contains less than three special menus) where the menu can be successfully subtyped. Note that classifying an operation as WE does not mean that every time this operation is executed the new system state will be consistent with the integrity constraints. For instance, if the system state where we apply the classifyAsSpecialmenu operation already contains three special menus, the operation will fail because of the integrity constraint $\operatorname{Cmax}($ SpecialMenu) $=3$, which defines the class Specialmenu may have at most three instances.

\subsubsection{Strongly Executable Operations}

We consider an operation is strongly executable (SE) if it is guaranteed to always generate a consistent state. That is to say, an operation is SE if it is always successfully executed, i.e. if every time we execute the operation (whatever values are given as arguments for its parameters), the effect of the actions included in the operation evolves the initial state of the system to a new state that satisfies all the integrity constraints of the structural model. Note that, unlike weak executability, strong executability requires all executions of the operation to be successful.

More formally:

Let $\mathrm{ExM}=\langle\mathrm{SM}, \mathrm{BM}\rangle$ be an executable model, an operation op $\in \mathrm{BM}$ is strongly executable (SE) iff $\forall \mathrm{p} \in \operatorname{Paths}(\mathrm{op}) \wedge \forall \mathrm{s}$ IsConsistent(s,SM) $\wedge \forall \mathrm{s}$ ' $\in$ AllExecutions $(\mathrm{p}, \mathrm{s})$ IsConsistent $\left(\mathrm{s}^{\prime}, \mathrm{SM}\right)$

Example 7 As we have seen, operation classifyAsSpecial$\mathrm{Menu}$ is not SE since after its execution we may violate the maximum cardinality integrity constraint of class specialmenu $(C \max ($ SpecialMenu $)=3)$, in particular, when the system state where the operation is applied already contains three special menus. Instead, operation addMenu is SE since we may guarantee it will never violate any integrity constraint of the structural model after 
their execution. Note that, in this case, the operation addMenu includes a guard (i.e. a precondition) to guarantee the changes this operation performs will only be executed in a safe context.

\subsubsection{Non Executable Operations}

Operations that are not WE are non executable $(\neg \mathrm{E})$. Non executable operations never generate a consistent system state. After their execution, they always reach a state of the system that violates some integrity constraints of the structural model (e.g. some cardinality constraints).

More formally:

Let $\mathrm{ExM}=\langle\mathrm{SM}, \mathrm{BM}\rangle$ be an executable model, an operation op $\in \mathrm{BM}$ is non executable $(\neg \mathrm{E})$ iff $\forall \mathrm{p} \in \operatorname{Paths}(\mathrm{op}) \wedge \forall \mathrm{s}$ IsConsistent(s,SM) $\wedge \forall \mathrm{s}^{\prime} \in$ $\operatorname{AllExecutions}(\mathrm{p}, \mathrm{s}) \neg \mathrm{Is} C$ Consistent $\left(\mathrm{s}^{\prime}, \mathrm{SM}\right)$

Example 8 Since newCourse is not WE, it is non executable.

\section{Verifying Executable Models}

In the previous section we have intuitively seen examples of executable and non-executable operations (see Examples 7-9). However, for general and complex operations, the manual reasoning to verify this property is tedious and error-prone. In this section we provide a lightweight and static method that automatizes this process to help the designers to verify her executable models.

Out method (see Figure 13) interacts with the designer through the following data:

- Input. The method takes as input an executable model composed by a structural model (a UML class diagram) and a behavioural model composed by a set of operations. The designer can select which operations from the behavioural model must be verified, that typically should be those that need to behave as a transaction. Since UML does not have a native support for transaction modeling, to mark an operation as transactional in UML, several existing approaches could be adapted to our needs, for instance: (1) the DSL presented in [32] to specify business and Web transactions; (2) an extension of the Ubiquitous Web Applications Transaction Design meta-model, which specifically addresses the design of Web application Transactions [18]; (3) UTML, a high level transaction design language to facilitate the complex web transaction design process [25]; or (4) the command pattern to encapsulate a request as an object, thereby letting you parameterize clients with different requests and support transactions as undoable operations [22].

- Output. For each selected operation, the method returns either a positive answer, meaning that the operation is WE/SE or a corrective feedback (see Section 5.4), consisting in a set of actions and guards that should be added to the operation in order to make it WE/SE. Note that, extending 
the operation with the provided feedback is a necessary condition but not a sufficient one to immediately guarantee the WE/SE of the operation since the added actions may in turn induce other constraint violations. Therefore, the extended operation must be iteratively reanalyzed with our method until we reach a WE/SE status.

When analyzing the WE/SE of an operation we must take into account all the possible execution paths (see Section 4): an operation is WE iff at least one of its execution paths is WE; and it is SE iff all its executions paths are SE; otherwise it is $\neg$ E. Therefore, prior to checking the weak/strong executability of an operation, our method performs a pre-processing step to compute its execution paths (Step 0). Once the execution paths have been computed, Steps 1 and 2 of the method are applied on each path $p$ until we recognize a WE path (in case of verifying weak executability) or until we check all paths are SE (in case of verifying strong executability). First, Step 1 (see Section 5.1) individually analyzes each action in the path $p$ to see whether it may violate some integrity constraints of the structural model. Then, Step 2 (see Section 5.2 ) performs a contextual analysis of each potentially violating action to see whether other actions or conditions in $p$ compensate or complement its effect to ensure that we sometimes/always reach a consistent state at the end of the operation execution. If all potentially violating actions can be discarded we can conclude that $p$ is WE/SE. Finally, Step 3 (see Section 5.3) classifies the operation depending on the results obtained in the previous step.

Our method performs an over-approximation analysis. Over-approximation is due to the lack of exhaustiveness in the comparison of conditions in the operation to favor the efficiency of the process. This implies that, in case of verifying the SE, our method may return false positives ${ }^{6}$, that is, it may return as a non SE an operation which is actually SE. On the other hand, the method does not return false negatives (in our opinion, more critical than the above), that is, when it states that an operation is SE, this statement is always true. As will be discussed in Section 8, this over-approximation may be eliminated either with the user intervention or using external methods.

In the following subsections we describe the three steps of our verification method (Sections 5.1, 5.2 and 5.3) and the feedback it provides (Section 5.4).

\footnotetext{
${ }^{6}$ According to the Oxford Dictionaries, we understand that a false positive is a test result which wrongly indicates that a particular condition or attribute is present (http://www.oxforddictionaries.com/definition/english/false-positive). Otherwise, a false negative is test result which wrongly indicates that a particular condition or attribute is absent (http://www.oxforddictionaries.com/definition/ english/false-negative). Then, when our method returns as a non WE/SE an operation which is actually WE/SE, we are wrongly indicating that the operation is incorrect, i.e. our method returns a false positive. Otherwise, if our method returned as a WE/SE an operation which was not actually WE/SE, it would wrongly indicate that the operation is correct, i.e. it would return a false negative.
} 


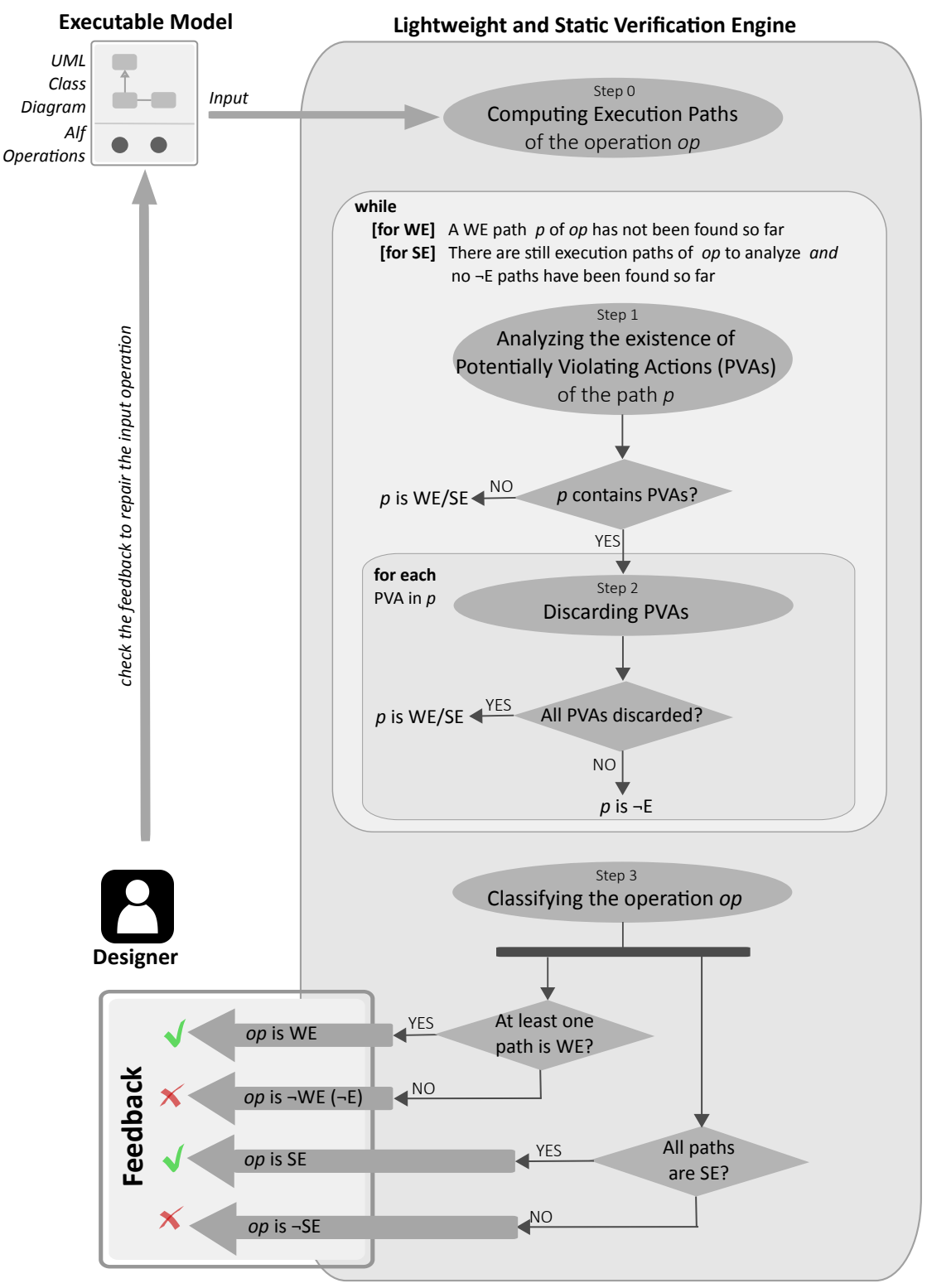

Figure 13: Method overview.

\subsection{Step 1: Analyzing the Existence of Potentially Violating Actions}

First step of our verification method analyzes each action in the path to see whether its effect can change the system state in a way that some integrity constraints of the structural model become violated. If so, this action is declared as Potentially Violating Action (PVA) and we refer to the constraints the PVA 
can violate as Susceptible Violated Constraints (SVC). If the path has no PVAs, it is WE/SE (and if we are checking if the operation is WE, we can directly confirm it at this step). Otherwise, we need to continue the analysis with the next step.

In order to detect the PVAs we have defined a set of rules that automatically determine the actions that may violate each integrity constraint of the structural model. Table 3 shows these rules. First column (Susceptible Violated Constraint $(S V C)$ ) shows each constraint our method supports (see them at Section 3) and second column (Potentially Violation Actions (PVAs)) determines the modification actions each constraint may violate. Several subrows for the same integrity constraint indicate several actions that may violate this constraint. Sharp sign (\#) represents irrelevant variables and consecutive letters $(\mathrm{x}, \mathrm{y}, \ldots)$ represent free variables that may be bound to any value in the action. Note that, when the minimum cardinality constraint of a class $(\mathrm{Cmin}(\mathrm{cl}))$ or of an association $(C \min (a s, r o l e))$ is not restricted (i.e. it is equal to zero), then no action may violate this constraint. Similarly, when the maximum cardinality constraint of a class $(C \max (\mathrm{cl}))$ or of an association $(C \max (\mathrm{as}, \mathrm{role}))$ is not restricted (i.e. it is equal to "*"), then no action may violate this constraint.

Table 3: Rules to determine the actions that may violate each integrity constraint.

\begin{tabular}{|c|c|c|}
\hline & $\begin{array}{l}\text { Susceptibler Vio- } \\
\text { lated Constraint } \\
(S V C)\end{array}$ & Potentially Violating Actions (PVAs) \\
\hline \multirow[t]{2}{*}{1} & \multirow[t]{2}{*}{$\operatorname{Cmin}(\mathrm{cl}) \neq 0$} & $\circ$. destroy (), where $\circ$ is an instance of class $\mathrm{cl}$ or of a subclass of $\mathrm{cl}$ \\
\hline & & $\begin{array}{l}\text { classify } \mathrm{x} \text { from } \circ \mathrm{ldCl} \text {, where } \circ \mathrm{ldCl} \text { includes the class } \mathrm{Cl} \text { or one of } \\
\text { its subclasses (only applies when } \mathrm{cl} \text { is child of a generalization) }\end{array}$ \\
\hline \multirow[t]{3}{*}{2} & \multirow[t]{3}{*}{$\operatorname{Cmax}(\mathrm{cl}) \neq^{*}$} & $\mathrm{x}=$ new $\mathrm{cl}()$ \\
\hline & & $\mathrm{x}=$ new $\mathrm{cl}^{\prime}()$, where $\mathrm{Cl}^{\prime}$ is a subclass of $\mathrm{cl}$ \\
\hline & & $\begin{array}{l}\text { classify } \times \text { to newCl, where newCl includes the class } \mathrm{Cl} \text { or one of } \\
\text { its subclasses (only applies when } \mathrm{Cl} \text { is child of a generalization) }\end{array}$ \\
\hline \multirow[t]{4}{*}{3} & \multirow[t]{4}{*}{ Mand(attr,cl) } & $\mathrm{x}=$ new $\mathrm{cl}()$ \\
\hline & & $\mathrm{x}=$ new $\mathrm{cl}^{\prime}()$, where $\mathrm{cl}^{\prime}$ is a subclass of $\mathrm{cl}$ \\
\hline & & $\begin{array}{l}\text { classify } \times \text { to newCl, where newCl includes the class } \mathrm{Cl} \text { or one of } \\
\text { its subclasses (only applies when } \mathrm{Cl} \text { is child of a generalization) }\end{array}$ \\
\hline & & $\begin{array}{l}\mathrm{x} . \text { attr }=\text { null, where } \mathrm{x} . \text { oclIsTypeof }(\mathrm{cl}) \text { or } \mathrm{x} . \text { oclIsTypeof }\left(\mathrm{cl}^{\prime}\right) \\
\text { and } \mathrm{cl}^{\prime} \text { is a subclass of } \mathrm{cl}\end{array}$ \\
\hline 4 & $\operatorname{Cmax}\left(\right.$ attr,cl) $\neq^{*}$ & $\begin{array}{l}0 . \operatorname{att} r=\# \text {, where } \circ \text { is an instance of the class } \mathrm{cl} \text { or of a subclass of } \\
\mathrm{cl}\end{array}$ \\
\hline \multirow[t]{4}{*}{5} & \multirow[t]{4}{*}{$\operatorname{Cmin}(a s, r) \neq 0$} & $\begin{array}{l}x=\text { new } \mathrm{cl}(), \text { where } \mathrm{cl} \text { (or one of its superclasses) participates on } \\
\text { the association as with role } r^{\prime}\left(r^{\prime} \text { is the opposite role to } r \text { in as) }\right.\end{array}$ \\
\hline & & $\begin{array}{l}\text { classify } \times \text { to newCl, where newCl includes the class } \mathrm{cl} \text { and } \mathrm{cl} \text { (or } \\
\text { one of its superclasses) participates on the association as with role } r^{\prime} \\
\text { ( } r^{\prime} \text { is the opposite role to } r \text { in as) (only applies when } \mathrm{cl} \text { is child of a } \\
\text { generalization) }\end{array}$ \\
\hline & & $\begin{array}{l}\text { as destroylink }(x, y) \text {, where the pair of objects }(x, y) \text { participate on } \\
\text { the association as }\end{array}$ \\
\hline & & $\begin{array}{l}\text { as.clearAssoc }(\circ) \text {, where } \circ \text { participates on the association as with } \\
\text { role } r^{\prime} \text { (and } r^{\prime} \text { is the opposite role to } r \text { in as) }\end{array}$ \\
\hline 6 & $\operatorname{Cmax}(\operatorname{as}, r) \neq *^{*}$ & as.createLink $(x, y)$ \\
\hline 7 & $\begin{array}{l}\operatorname{Cov}\left(\mathrm{cl},\left\{c l_{1}, \ldots,\right.\right. \\
\left.\left.c l_{n}\right\}\right)\end{array}$ & classify $x$ from $\circ l d C l$, where $\circ l d C l$ includes one $c l_{i}$ \\
\hline 8 & $\begin{array}{l}\operatorname{Disj}\left(c l,\left\{c l_{1}, \ldots,\right.\right. \\
\left.\left.c l_{n}\right\}\right)\end{array}$ & classify $x$ to newCl, where newCl includes one $c l_{i}$ \\
\hline 9 & $\mathrm{ID}($ attr,cl) & $\begin{array}{l}\circ \cdot \operatorname{attr}=\#, \text { where } \circ \text { is an instance of the class } \mathrm{cl} \text { or of a subclass of } \\
\mathrm{cl}\end{array}$ \\
\hline \multirow[t]{2}{*}{10} & \multirow[t]{2}{*}{ Sym(as) } & as.createLink $(\mathrm{x}, \mathrm{y})$ \\
\hline & & as.destroyLink $(\mathrm{x}, \mathrm{y})$ \\
\hline & & Continued on next page \\
\hline
\end{tabular}


Table 3 - continued from previous page

\begin{tabular}{|c|c|c|}
\hline & $\begin{array}{lr}\text { Susceptible Vio- } \\
\text { lated Constraint } \\
(S V C)\end{array}$ & Potentially Violating Actions (PVAs) \\
\hline 11 & Asym(as) & as.createLink $(\mathrm{x}, \mathrm{y})$ \\
\hline 12 & Irrefl(as) & as.createlink $(x, x)$ \\
\hline 13 & $\begin{array}{l}\text { ValueComp } \\
\text { (attr,op,v) }\end{array}$ & $\begin{array}{l}\circ \text { attr }=\#, \text { where } \circ \text { is an instance of the class which owns attr or } \\
\text { of one of its subclasses }\end{array}$ \\
\hline 14 & Referential(cl,as $)^{7}$ & $\begin{array}{l}\text { classify } \circ \text { from } \circ \mathrm{ddCl} \text {, where } \circ . \circ \mathrm{cl} \text { IsType } \circ(\mathrm{cl}) \text { (before classi- } \\
\text { fying } \circ \text { ), } \circ \mathrm{ldCl} \text { includes the class } \mathrm{cl} \text { and } \mathrm{cl} \text { participates on the asso- } \\
\text { ciation as (only applies when } \mathrm{cl} \text { is child of a generalization) }\end{array}$ \\
\hline
\end{tabular}

As an example, we discuss the first row of Table 3, which determines the actions that may violate the minimum cardinality constraint of a class $c l$ when it is different to zero $(\operatorname{Cmin}(\mathrm{cl}) \neq 0)$ :

- First subrow indicates every time we destroy an object of class cl or of a subclass of $\mathrm{Cl}$ (that is, the number of instances of $\mathrm{cl}$ is decreased), we may violate the constraint $C \min (\mathrm{cl})$.

- Similarly, second subrow indicates every time we take out an object from class $\mathrm{cl}$ or from one of its subclasses, we also may violate this constraint.

In this first step, the rules of Table 3 are applied over all the integrity constraints that appear in the input structural model. As a result, we obtain the set of potentially violating actions (PVAs) that may violate each integrity constraint of the structural model. Then, we may determine whether a path $p$ contains PVAs by comparing this set of actions with the set of actions which appear in $p$. All actions in the intersection of both sets are PVAs.

In order to do this comparison a mapping between the PVAs obtained from Table 3 and the actions of the path has to be done. An action of the first set (cointaining generic PVAs) can be mapped onto an action of the second set (containing specific PVAs obtained from the operation paths) when the following conditions are satisfied: (1) both actions are from the same type (e.g. CreateObject, ReclassifyObject, etc.); (2) the model elements referenced by the actions coincide (e.g. both CreateObjects create objects of the same class); and (3) all instance-level parameters of the generic PVA (i.e. variables $\mathrm{x}, \mathrm{y}, \ldots$ ) can be bound to the parameters of the specific PVA (irrelevant variables - i.e. \# - may be bound to any parameter value in the specific PVA).

Example 9 As an example, we show the PVAs for the two execution paths of operation newCourse. Second path (see Figure 14, where PVAs are highlighted in red) contains two PVAs: (1) the 1st action in the path, which may violate two mandatory constraints (when the attributes description and category are not initialized); and (2) the last action in the path, which may violate the symmetric

\footnotetext{
${ }^{7}$ Note that this constraint is not violated when we destroy an object of type cl, because the Alf semantics for the action DestroyObject ensures the destruction of all links in which the destroyed object participates.
} 
association constraint (when the opposite link is not created) and the irreflexive association constraint (when the link connects an object with itself). First path (which is a subset of the former) only contains the first PVA. Then, in order to determine if these paths are $\mathrm{WE} / \mathrm{SE}$, we need to continue the analysis with the next step.

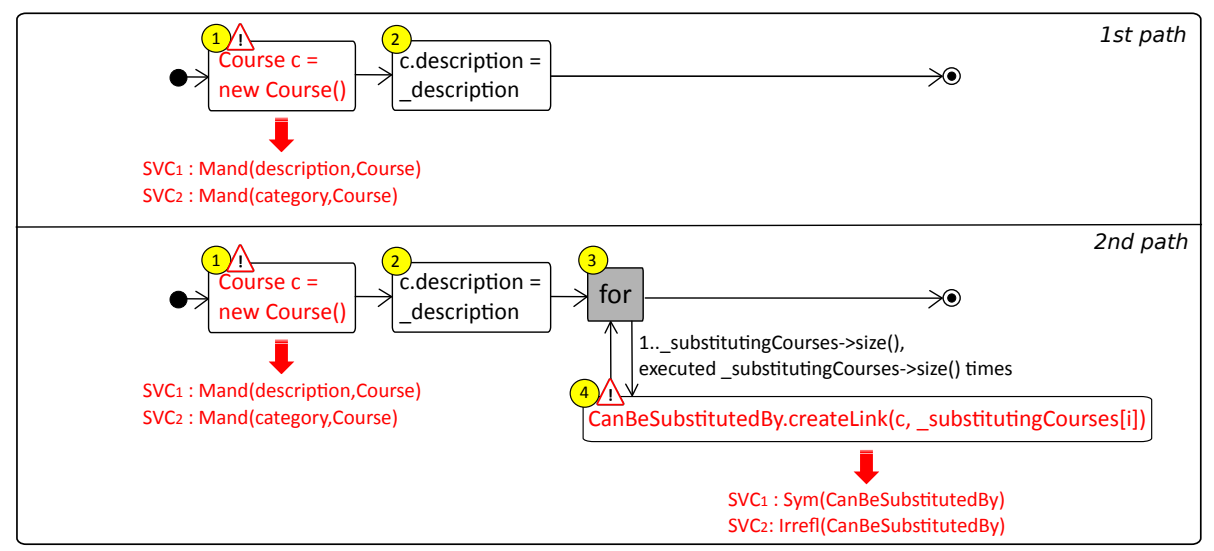

Figure 14: PVAs of the paths of the operation newCourse.

\subsection{Step 2: Discarding Potentially Violating Actions}

It may happen that the context in which a PVA is executed within the path guarantees that the effect of the PVA is not going to actually violate any of its SVCs. In these cases, the PVA may be discarded. Roughly, there are two ways to discard a PVA: (1) when the path contains a guard (i.e. a precondition) that ensures the PVA will only be executed in a safe context; and (2) when the path contains another action which counters or complements the effect of the PVA in order to maintain the integrity of the system after executing the operation.

In this second step, our method analyzes each PVA returned by the previous step and tries to discard them by analyzing the above possibilities. If all PVAs that may compromise the WE/SE of the path can be discarded, then it is classified as WE/SE. If not, the path is marked as $\neg \mathrm{WE} / \neg \mathrm{SE}$ and the corresponding corrective feedback is provided (see Section 5.4). Note that, if a PVA may violate several SVCs, it may be discarded only when it satisfies all the conditions to avoid violating each SVC.

The conditions to discard the PVAs are expressed as action patterns that should be matched in the path. To the sake of simplicity, here we only show a small subset of the forty patterns we designed (the complete catalog of patterns can be found on [50]). They are shown in Table 4, which has several columns:

- PVA: States the PVA we are trying to discard.

- $S V C$ : States the constraint the PVA may violate. 
- Conditions to discard the PVA: Describes the conditions the path must satisfy to discard the PVA in order to avoid the violation of the SVC. Conditions are expressed as an Alf pattern, i.e. in reference of Alf statements that the path should or should not include complemented with a textual description.

Note that when trying to match guard expressions (for instance, conditional structures) we follow a syntactic approach, i.e. we do not try to formally prove the expression in the path implies the expression in the guard (which would be too costly for general expressions) but just to check whether a path expression matches one of the syntactic variation patterns predefined for the condition. For instance, if our pattern is expecting the guard cl.allinstances () $\rightarrow$ size ()$<x$ (where $x$ is an integer) and the checked operation contains the guard cl.allinstances()->size ()<OCLexpr (where OCLexpr is a new OCL expression instead an integer), our algorithm does not semantically compares $\mathrm{x}$ and OCLexpr but concludes the two guards do not syntactically match. When the algorithm cannot conclude the implication it assumes that one does not imply the other. This is why the method over-approximates the results (as a necessary trade-off to foster the efficiency of the method) as commented previously. The designer could optionally participate in this step to manually identify those implications that were not found by the method using its syntactic approach.

Table 4: Conditions to discard PVAs.

\begin{tabular}{|c|c|c|c|c|}
\hline & PVA & SVC & \multicolumn{2}{|c|}{ Conditions to discard the PVA } \\
\hline \multirow[t]{2}{*}{1} & \multirow[t]{2}{*}{$o=$ new $c l()$} & \multirow[t]{2}{*}{ Mand(attr,cl) } & Pattern & $\begin{array}{l}\circ=\text { new } c l() ; / / \text { PVA } \\
\cdots \\
\text { o.attr }=\# ;\end{array}$ \\
\hline & & & Descr. & $\begin{array}{l}\text { The path includes, after the PVA, } \\
\text { at least one action to initialize the } \\
\text { attribute } a t t r .\end{array}$ \\
\hline \multirow[t]{2}{*}{2} & \multirow[t]{2}{*}{$\circ=$ new $\mathrm{cl}()$} & \multirow[t]{2}{*}{$\operatorname{Cmin}(a s, r) \neq 0$} & Pattern & 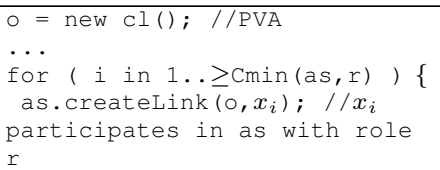 \\
\hline & & & Descr. & $\begin{array}{l}\text { The path includes, after the PVA, at } \\
\text { least Cmin (as, r) actions to create a } \\
\text { link of as between the new object o } \\
\text { and another object (with role } r \text { ). }\end{array}$ \\
\hline \multirow[t]{3}{*}{3} & \multirow[t]{3}{*}{ as.createLink $\left(o_{1}, o_{2}\right)$} & \multirow[t]{2}{*}{ Sym(as) } & Pattern & $\begin{array}{l}\text { as.createLink }\left(o_{1}, o_{2}\right) ; / / \text { PVA } \\
\text {... } \\
\text { as.createLink }\left(o_{2}, o_{1}\right) ;\end{array}$ \\
\hline & & & Descr. & $\begin{array}{l}\text { The path includes the creation of } \\
\text { the symmetric link. }\end{array}$ \\
\hline & & Irrefl(as) & Pattern & $\begin{array}{l}\text { if }\left(o_{1} \neq o_{2}\right)\{ \\
\text { as.createLink }\left(o_{1}, o_{2}\right) ; / / \mathrm{PVA} \\
\ldots \\
\text { J } \\
\text { The path contains a guard that } \\
\text { prevents the execution of the PVA } \\
\text { when the two member ends are the } \\
\text { same object. }\end{array}$ \\
\hline
\end{tabular}


Example 10 As an example, we try to discard the PVAs of the second execution path of the operation newCourse (note that the first path is a subset of the former). As we justified in the previous step, this path contains two PVAs: (1) the first action: Course $\mathrm{c}=$ new Course( ) and (2) the last: CanBeSubstitutedBy . createLink (c, substitutingCourses [i]). According to 1st row of Table 4, in order to discard the first PVA (see action 1 of Figure 15) when it may violate a constraint of type mandatory, the path must include, after the PVA, at least one action to initialize the attributes description and category. The path contains an action (see action 2 of Figure 15) to initialize the attribute description but it does not contain any action to initialize the attribute category. Then the first PVA cannot be discarded because it always violate the constraint Mand(category, Course).

At this point, our method can conclude this path is $\neg \mathrm{E}$ since it always violates the above constraint. However, in order to illustrate a complete example, in the following we analyze the remaining PVAs.

According to 3rd row of Table 4 (1st subrow), in order to discard the second PVA (see action 4 of Figure 15) when it may violate a constraint of type symmetric, the path must include the creation of the symmetric link. The path does not create this link. Besides, according to 3rd row of Table 4 (2nd subrow), in order to discard the same PVA when it may violate a constraint of type irreflexive, the path must include a guard to prevent the execution of the PVA when the two courses are the same object. The path does not include this guard. Then the second PVA cannot be discarded and, as we pointed out before, our method concludes this path is not WE/SE.

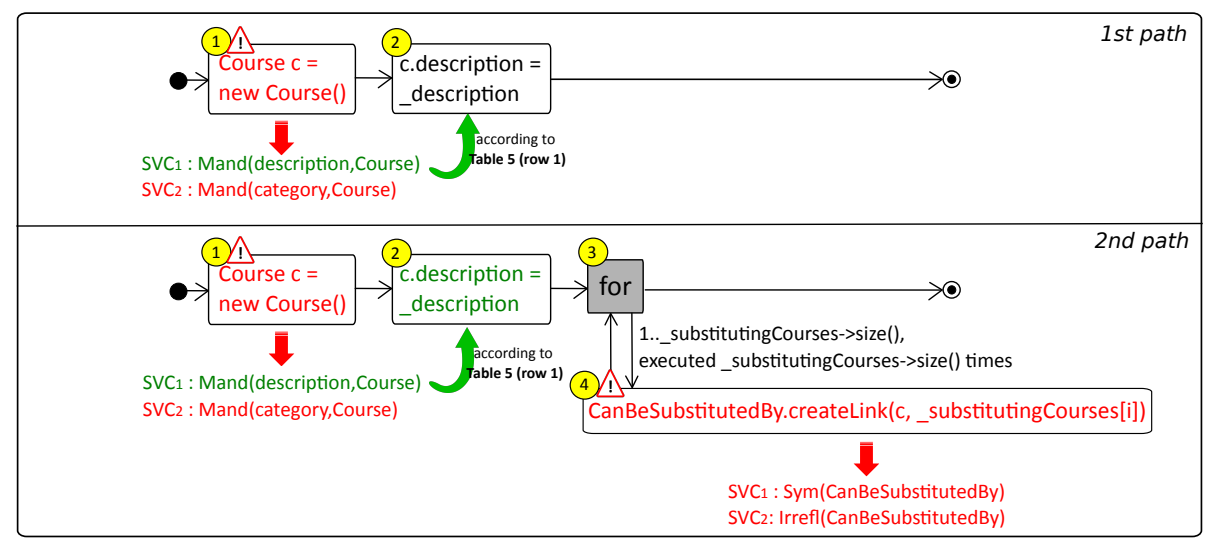

Figure 15: Discarding PVAs for the second path of newCourse operation. 


\subsection{Step 3: Classifying the Operation}

Last step of our method classifies the operation depending on the results obtained in the previous step regarding each execution path of the operation.

If at least one of the execution paths of the operation is WE, the operation is classified as WE. If all its execution paths are $\mathrm{SE}$, the operation is classified as SE. Otherwise, the operation is classified as $\neg \mathrm{E}$.

Example 11 Since, as shown previously, both paths of the newCourse operation are neither WE nor SE, our method concludes this operation is $\neg \mathrm{E}$. Next section shows how to correct this.

\subsection{Feedback}

Besides determining the executability of an operation, a distinguishing feature of our method is that for $\neg \mathrm{WE} / \neg \mathrm{SE}$ operations it returns valuable information to help the designers identifying and correcting the detected errors. This feedback information is expressed in terms of the operation itself so it can be easily understood and processed by the designer.

For $\neg \mathrm{WE} / \neg \mathrm{SE}$ operations, our method provides two kinds of information: (1) the returned feedback identifies why the operation is non executable, i.e. for each $\neg \mathrm{WE} / \neg \mathrm{SE}$ path our method provides the list of PVAs that could eventually induce a violation of the integrity constraints together with the specific list of SVCs that those PVAs could violate; and (2) the returned feedback explains how the designer may fix these (potentially) violating scenarios by providing a set of possible repair alternatives that should be included in the $\neg \mathrm{WE} / \neg \mathrm{SE}$ operation paths. These alternatives are expressed as a finite set of Alf-patterns (see the complete catalog on [50]) to be added to the path of those PVAs that cannot be discarded. The designer should choose the most appropriate alternative in her context.

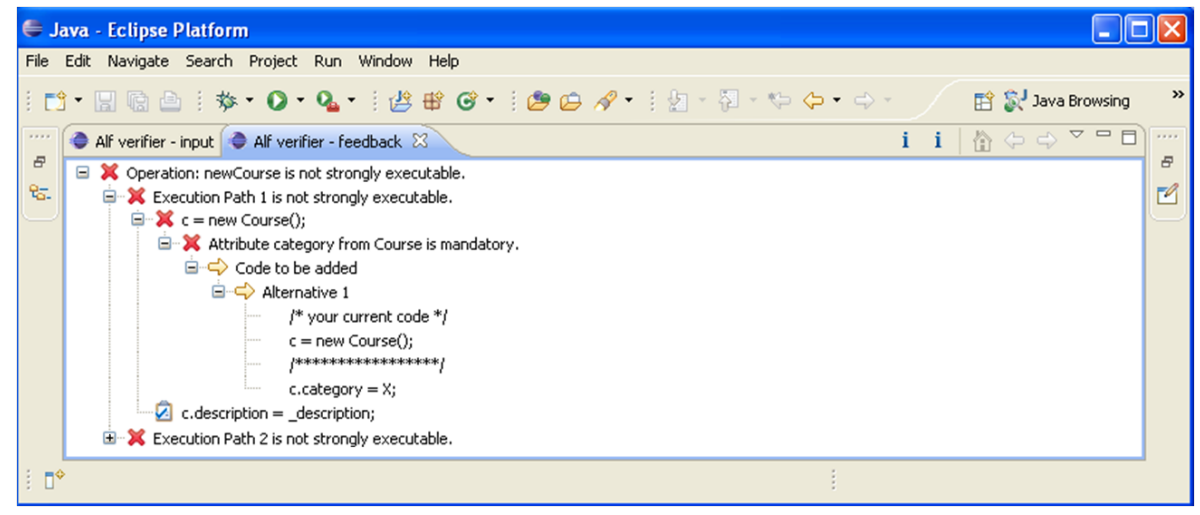

Figure 16: Feedback for newCourse operation. 
Example 12 Figure 16 shows the feedback provided when verifying the strong executability for the operation newCourse. Next, we show the repaired operation once the feedback provided by our method has been integrated. The added sentences are emphasized in bold type. Each added sentence fixes one of the problems detected in the previous section.

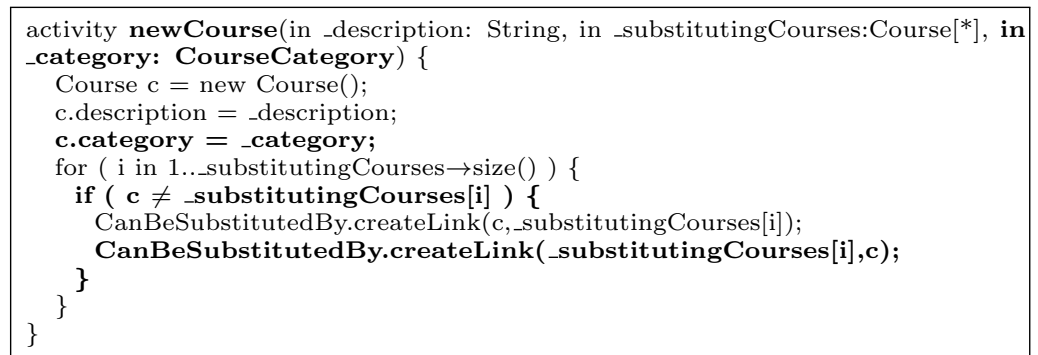

Three changes have been applied to the operation newCourse: (1) the initialization of the attribute category, which ensures the constraint Mand(category, Course) will never be violated; (2) the guard, which ensures the constraint type irreflexive will never be violated; and (3) the creation of a new link, which ensures the constraint Sym(CanBeSubstitutedBy) will never be violated. After applying these changes, the operation newCourse becomes WE and SE.

\section{Tool Support}

In order to prove the feasibility of our method, we have built a prototype tool that implements our algorithm for verifying Alf operations. The tool has been implemented as an Eclipse plug-in which can be downloaded from [1]. A demonstration video about it can be viewed on [2].

Figure 17 shows the general view of the tool architecture. As a first step, the designer specifies the UML executable model she wants to deal with. The structural model, composed by a UML class diagram and a set of OCL integrity constraints, is modelled using the graphical modelling environment provided by UML2Tools [59], an Eclipse Graphical Modeling Framework for manipulating UML models. The behavioural model, composed by a set of Alf operations, is specified in a text file with .alf extension. Once both models have been defined, the designer selects the operation/s and the property (weak or strong executability) she wants to verify. Then, the core of our method is invoked to perform the static analysis we have described in Section 5. Finally, the feedback provided by our method is displayed, integrated into the Eclipse interface. Once the designer receives the feedback she can (manually) discard those implications that cannot be detected by our method and/or (manually) modify the operation according to the provided feedback to fix the detected errors.

Internally, our tool is implemented as a set of Java classes extended with the library of the UML2Tools [59] to interact with the input UML model (i.e. 


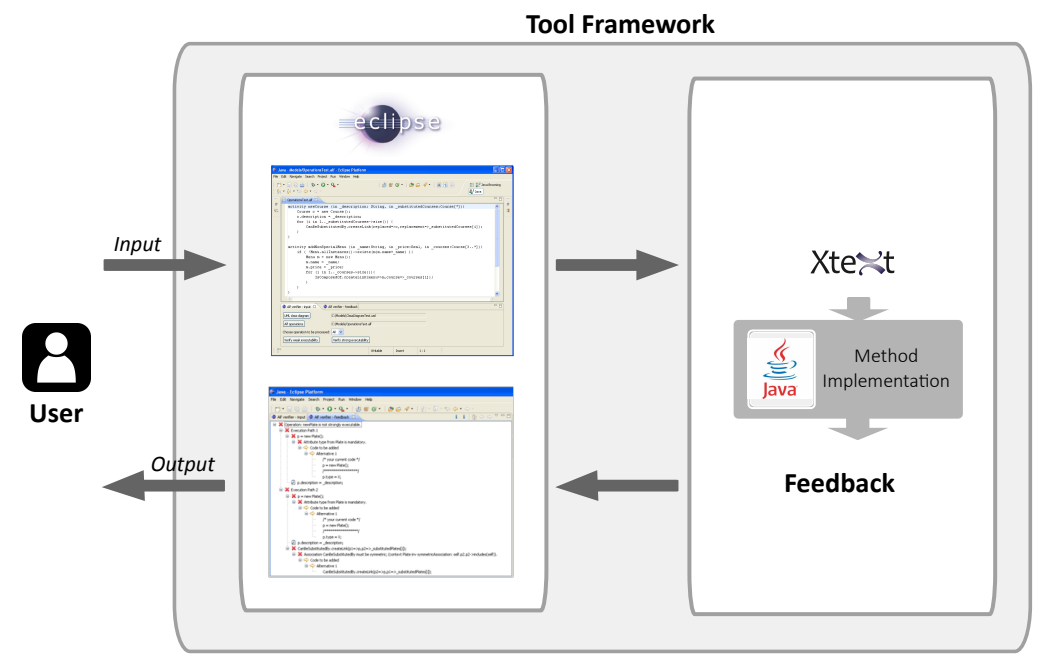

Figure 17: General architecture or our tool.

the class diagram and its constraints). To parse the input Alf operations, we have implemented an Alf parser using the Xtext framework [3]. To this end, we depart from the Alf grammar (defined using an EBNF notation) and use the Abstract Syntax Tree provided by Xtext to generate the Java classes that instantiate the classes of the fUML metamodel.

As an example, Figure 18 shows the main form of our Eclispe plug-in, which permits to import both the UML model and the Alf operations and to choose operation/s and the property the designer wants to verify. A screenshot of the feedback for the operation newCourse provided by our method has been shown in Figure 16.

This prorotype tool is a first attempt to implement our method so it could be extended in several ways (see Section 10).

\section{Empirical Evaluation}

We evaluated our method concerning its usefulness and its efficiency. In the following we present two experiments we carried out to evaluate the above features.

\subsection{Usefulness of Our Method}

The first experiment was aimed to evaluate the usefulness of our method, understanding the usefulness as the help for detecting and correcting defects in operations.

With this experiment, that complements the experiment presented in Section 2 , we compared the correctness of a model developed without using any verification method wrt the correctness of a model developed using our verification method. 


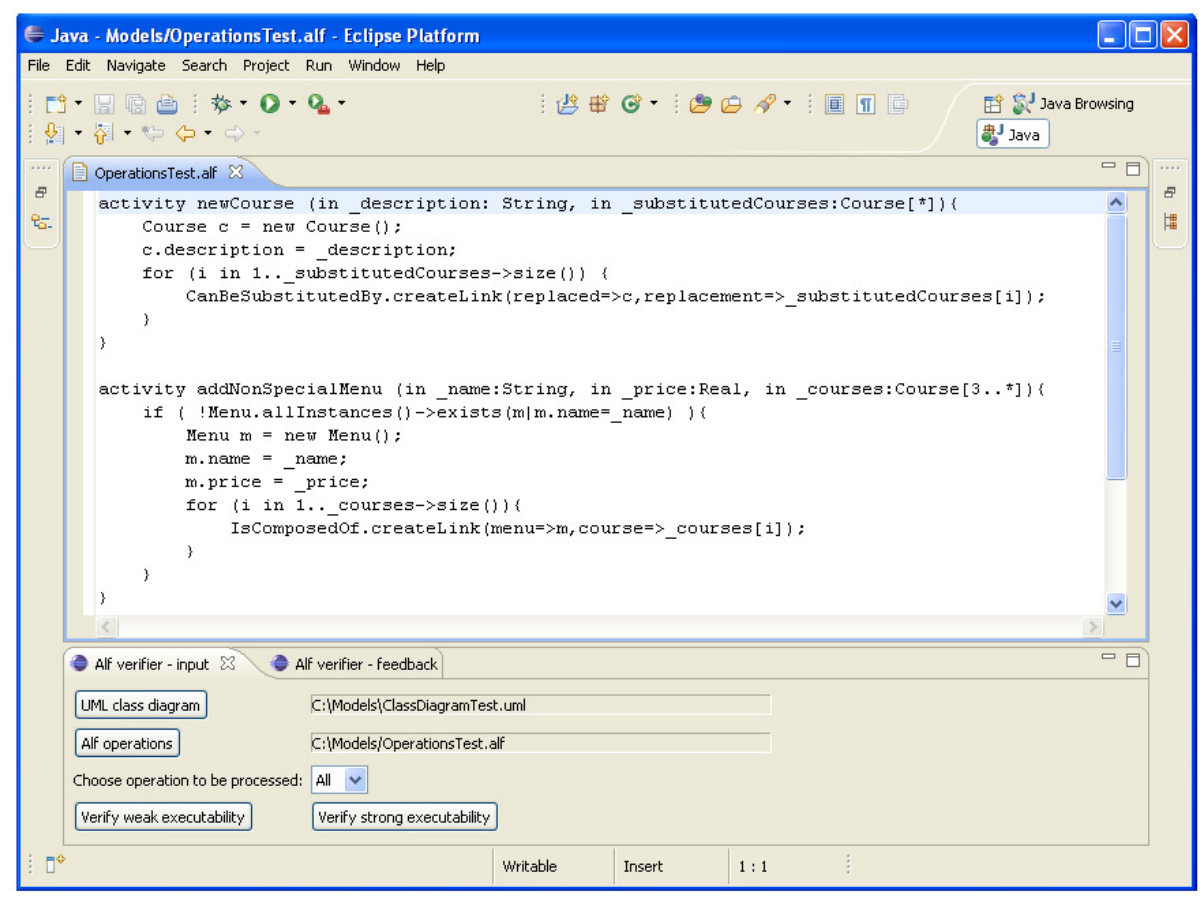

Figure 18: Screenshot of the input view.

A total of 124 students of two Software Engineering courses of the Computer Science Degree taught at the Open University of Catalonia (UOC) participated in the experiment. All of them had a good background about modeling and programming.

Before starting the experiment, the students were introduced to executability property using the running example presented in this paper. As a starting point, and to facilitate the understandability of the model, the students were provided with a simplified version of the class diagram shown in Figure 4 (containing 3 classes, 2 associations and 5 integrity constraints) and two operations of low complexity in terms of the number of actions they contained (an average of 4 actions each operation). Both operations were $\neg \mathrm{E}$ : the former violated one integrity constraint and the later violated two integrity constraints of the structural model. Then, two tasks were assigned to the students. First, they were encouraged to identify the source of the problem and correct both operations without using any help. Finally, and to conclude the experiment, the same students were encouraged to correct the same operations using the feedback provided by our method.

The results (see Figure 19) show that, when the students did not have any help, only $45 \%$ of them were able to properly correct the operations. All students that were not able to correct the operations neither were capable to identify the source of the problem in the operations. However, when the students were 
helped with the feedback provided by our method, $93 \%$ of them were able to correct the operations.

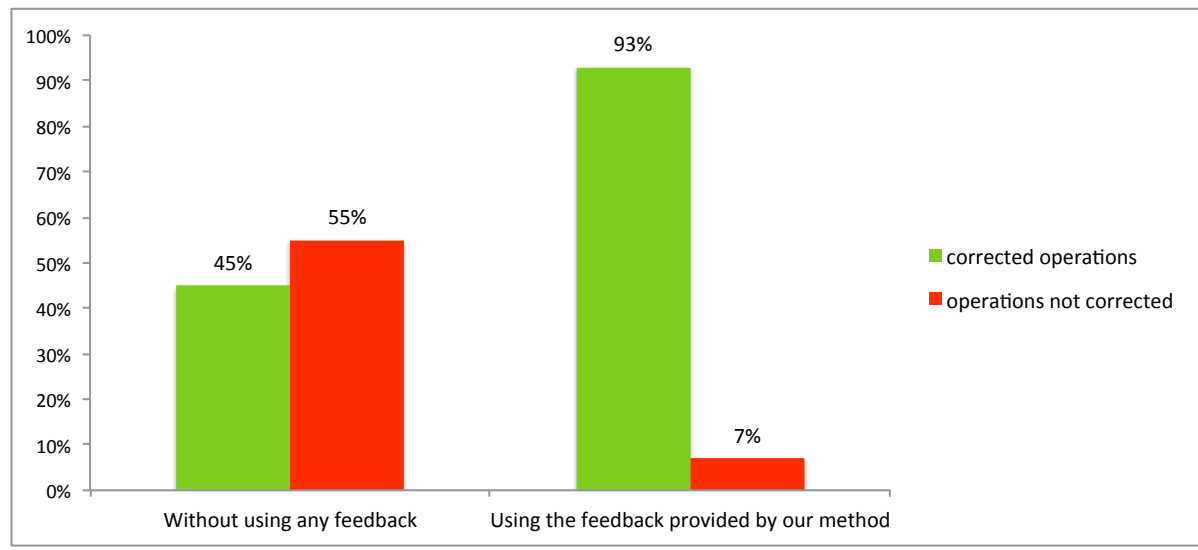

Figure 19: Results of our experiment.

We believe this experiment clearly justifies the usefulness that our method can bring to designers during the specification of their behavioural models.

\subsection{Efficiency of Our Method}

The second experiment was aimed to evaluate the efficiency of our method, understanding the efficiency as the capability to perform the verification in a reasonable time.

Given that current approaches consider different input models, properties and types of feedback, it is difficult to make a fair comparison of efficiency between our method and other approaches. Instead we aim to show that our method provides the appropriate feedback in a reasonable time.

In this second experiment we conducted several simulations with our Eclipse plug-in to measure the running time when verifying the strong executability of several operations. Note that, to verify this property all execution paths have to be analyzed, then, the time complexity when verifying the strong executability will be greater than the time complexity when verifying the weak executability (since in the former we must apply the method over all the paths of the operation while in the second we must apply the method until we reach a WE path).

The performance results for the simulations are detailed in Table 5, which contains several columns:

- Operation: contains the name of the verified operation. We have verified the three operations of our running example plus several test operations designed for this experiment.

- Class Diagram size: describes the size of the structural model regarding the number of classes, attributes, associations and generalizations appearing in the Class Diagram. 
- Number of Integrity Constraints: states the number of integrity constraints (considering those graphically represented in the class diagram plus OCL constraints).

- Operation size: describes the operation size in terms of the number of actions, loops and conditional structures it contains.

- Number of Execution Paths: states the total number of executions paths of the operation without considering the emtpy paths.

- Total number of PVAs: describe the total number of PVAs considering all the paths. It means that, if one PVA appears in two execution paths, it is counted twice since it will be considered during the analysis of both paths. This is the reason why in some cases an operation contains more PVAs than actions.

- Running time: time took for our method to perform the verification.

As can be seen in Figure 20, the time it took to perform the verification reasonably increases when one or more variables (number of integrity constraints, number of execution paths and/or number of PVAs appearing on those paths) rises. However, the above simulations show that all the simulations took less than 7 seconds. We consider this is a reasonable time for a verification, considering that the verification process will be launched by the user request (probably at the end of the design phase) and not automatically in each update.
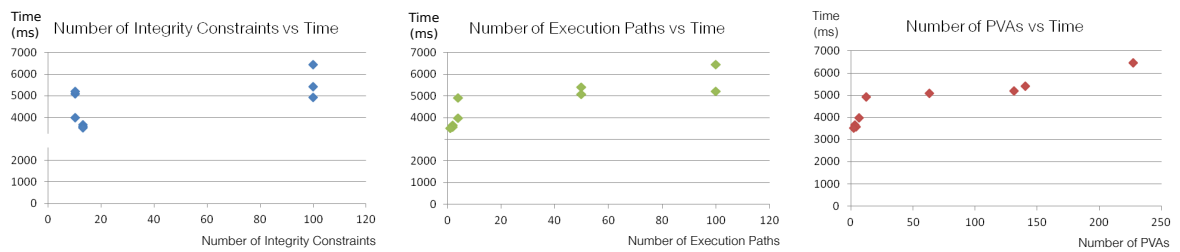

Figure 20: Results of our experiment.

Even though, theoretically, the computational cost of our method is exponential (wrt the size of the MBCFG) in the worst case (when all the actions of the operation are PVAs and none of them may be discarded), it is not directly affected by the size of the structural model (i.e. the number of classes, attributes, integrity constraints, etc). For this reason, and considering the above experiment, we consider our method is efficient.

\subsection{Reliability of Our Method}

The last experiment was aimed to evaluate the reliability of our method, understanding the reliability as the proportion of truth in the results provided by our method.

As we introduced, one limitation of our method is the fact that, when verifying the strong executability, it may return false positives (see Section 5). Section 
Table 5: Performance results for our prototype tool.

\begin{tabular}{|c|c|c|c|c|c|c|}
\hline Operation & $\begin{array}{l}\text { Class Dia- } \\
\text { gram size }\end{array}$ & $\begin{array}{l}\text { Number } \\
\text { of in- } \\
\text { tegrity } \\
\text { con- } \\
\text { straints }\end{array}$ & $\begin{array}{l}\text { Operation } \\
\text { size }\end{array}$ & $\begin{array}{l}\text { Number } \\
\text { of (non- } \\
\text { empty) } \\
\text { execution } \\
\text { paths }\end{array}$ & $\begin{array}{l}\text { Total } \\
\text { number } \\
\text { of PVAs }\end{array}$ & $\begin{array}{l}\text { Running } \\
\text { time }\end{array}$ \\
\hline newCourse & $\begin{array}{ll}6 \text { classes, } & 8 \\
\text { attributes, } & 4 \\
\text { associations, } & 1 \\
\text { generalization }\end{array}$ & 13 & $\begin{array}{l}3 \text { actions, } \\
1 \text { loop }\end{array}$ & 2 & 3 & $3641 \mathrm{~ms}$ \\
\hline addMenu & $\begin{array}{ll}6 \text { classes, } & 8 \\
\text { attributes, } & 4 \\
\text { associations, } & 1 \\
\text { generalization }\end{array}$ & 13 & $\begin{array}{l}4 \text { actions, } \\
1 \text { condi- } \\
\text { tional, } 1 \\
\text { loop }\end{array}$ & 2 & 4 & $3579 \mathrm{~ms}$ \\
\hline $\begin{array}{l}\text { classifyAs- } \\
\text { Specialmenu }\end{array}$ & $\begin{array}{ll}6 \text { classes, } & 8 \\
\text { attributes, } & 4 \\
\text { associations, } & 1 \\
\text { generalization }\end{array}$ & 13 & $\begin{array}{l}2 \text { actions, } \\
1 \text { condi- } \\
\text { tional }\end{array}$ & 1 & 2 & $3522 \mathrm{~ms}$ \\
\hline $\begin{array}{l}\text { scalability- } \\
\text { Test1 }\end{array}$ & \begin{tabular}{lr}
\multicolumn{1}{c}{ classes, } & 10 \\
attributes, & 4 \\
associations, & 1 \\
generalization
\end{tabular} & 10 & $\begin{array}{lr}10 \quad \text { ac- } \\
\text { tions, } & 1 \\
\text { condi- } & \\
\text { tionals, } & 1 \\
\text { loop } & \\
\end{array}$ & 4 & 6 & $3981 \mathrm{~ms}$ \\
\hline $\begin{array}{l}\text { scalability- } \\
\text { Test } 2\end{array}$ & $\begin{array}{l}100 \quad \text { classes, } \\
200 \text { attributes, } \\
40 \quad \text { associ- } \\
\text { ations, } \\
\text { generalizations }\end{array}$ & 100 & $\begin{array}{lr}10 \quad \text { ac- } \\
\text { tions, } & 1 \\
\text { condi- } & \\
\text { tionals, } & 1 \\
\text { loop } & \\
\end{array}$ & 4 & 12 & $4921 \mathrm{~ms}$ \\
\hline $\begin{array}{l}\text { scalability- } \\
\text { Test } 3\end{array}$ & $\begin{array}{lr}5 \text { classes, } & 10 \\
\text { attributes, } & 4 \\
\text { associations, } & 1 \\
\text { generalization }\end{array}$ & 10 & $\begin{array}{lr}100 & \text { ac- } \\
\text { tions, } & 10 \\
\text { loops, } & 10 \\
\text { condi- } & \\
\text { tionals } & \\
\end{array}$ & 50 & 63 & $5087 \mathrm{~ms}$ \\
\hline $\begin{array}{l}\text { scalability- } \\
\text { Test } 4\end{array}$ & $\begin{array}{l}100 \text { classes, } \\
200 \text { attributes, } \\
40 \quad \text { associ- } \\
\text { ations, } \\
\text { generalizations }\end{array}$ & 100 & $\begin{array}{lr}200 & \text { ac- } \\
\text { tions, } & 10 \\
\text { loops, } & 10 \\
\text { condi- } & \\
\text { tionals } & \\
\end{array}$ & 50 & 140 & $5419 \mathrm{~ms}$ \\
\hline $\begin{array}{l}\text { scalability- } \\
\text { Test5 }\end{array}$ & \begin{tabular}{lr}
\multicolumn{1}{c}{ classes, } & 10 \\
attributes, & 4 \\
associations, & 1 \\
generalization
\end{tabular} & 10 & $\begin{array}{lr}200 & \text { ac- } \\
\text { tions, } & 10 \\
\text { loops, } & 10 \\
\text { condi- } & \\
\text { tionals } & \\
\end{array}$ & 100 & 131 & $5211 \mathrm{~ms}$ \\
\hline $\begin{array}{l}\text { scalability- } \\
\text { Test } 6\end{array}$ & $\begin{array}{l}100 \text { classes, } \\
200 \text { attributes, } \\
40 \quad \text { associ- } \\
\text { ations, } 10 \\
\text { generalizations }\end{array}$ & 100 & $\begin{array}{lr}200 & \text { ac- } \\
\text { tions, } & 10 \\
\text { loops, } & 10 \\
\text { condi- } & \\
\text { tionals } & \\
\end{array}$ & 100 & 227 & $6462 \mathrm{~ms}$ \\
\hline
\end{tabular}


8 discusses about this fact. In this experiment we analyze the proportion of false positives returned by our method.

Although there is a lack of Alf models publicly available, we based our experiment in a total set of 30 Alf operations from several resources:

- 3 operations from the running example of this paper (with an average of 3 actions/operation), related to a class diagram composed by 6 classes, 8 attributes, 4 associations, 1 generalization and 13 integrity constraints.

- 12 operations of the OnlineBookStore case study [57] (with an average of 1.7 actions/operation), related to a class diagram composed by 6 classes, 12 attributes, 5 associations and 18 integrity constraints.

- 15 operations of the PropertyManagement case study [57] (with an average of 4.8 actions/operation), related to a class diagram composed by 6 classes, 61 attributes, 5 associations, 1 generalization and 68 integrity constraints.

After verifying the strong executability of the above operations, we found that our method was capable to provide the right result in 29 of the 30 executions. This means our method returns the truth in the $96.67 \%$ of the cases (i.e. it returns a false positive only in $3.32 \%$ of the cases). For this reason, and considering the above experiment, we consider the level of reliability of our method is acceptable.

\section{Discussion}

In this section we expose the assumptions our method relies on and discuss their limitations in order to evaluate its pros and cons.

\subsection{Assumptions of Our Method}

Our method assumes all Alf operations are syntactically correct (i.e. they conform to the standard Alf language [45]) and always terminate. This is a reasonable assumption and necessary to begin our analysis.

According to the widely accepted criteria about the elimination of the unreachable code [15], our method also assumes the body of all conditional and loop structures is reachable (given the proper input values). This means that the condition of all conditional and loop structures may be satisfied (i.e. they can evaluate to true) and then the body of these structures may be executed. This assumption guarantees all the actions within an operation can be executed. Otherwise (see Example 14), the actions in those paths that may be needed to compensate the effect of a PVA could not be used and thus falsify the results of the method. Roughly, this SAT-problem could be tackled with UML/OCL verification tools [14] adding the test condition as an additional constraint to the model and checking if the extended model is still satisfiable. However, this analysis, would worsen the efficiency of our method. 
Example 13 The code of the following operation is unreachable since the condition of the conditional structure always evaluate to false.

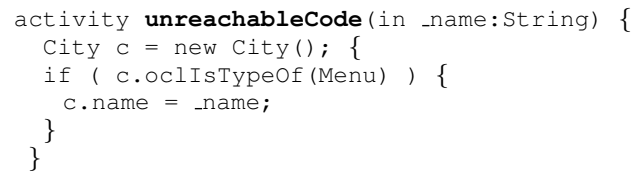

We also assume that operations to be analyzed do not include recursive invocations. This assumption is made because of recursive invocations generate infinite paths (given that the recursive invocation is replaced by the sub-diagraph corresponding to the operation itself, then, this replacement process never finishes) that are not able to be addressed by our method. Nevertheless, recursive operations could be transformed into their iterative counterparts [6] before the application of our method.

Finally, since we consider operations are defined by actions, we ignore other types of ActivityNodes (such as ControlNodes) and thus actions in the operation specification are always executed sequentially. The target platform could allow simultaneous execution of the same or different operations by the same or several users, which could introduce some inconsistencies on the data if not treated properly. Still, since the focus of our method is to verify single operations at design time, we can avoid the possible concurrency issues that could occur when several sequentially operations are concurrently executed in a specific context. This potential problem should be addressed by the execution platform itself (e.g. ensuring ACID properties, like in database systems, if necessary) and not responsibility of the individual operations themselves.

\subsection{Limitations of Our Method}

Moreover, our method presents several trade-offs that are required to enable our lightweight analysis.

As we noted before, one limitation of our method is the fact that it performs an over-approximation analysis. This implies that, when verifying the strong executability, our method may classify as a $\neg \mathrm{SE}$ an operation which is actually SE (but not the other way round, the method never marks as SE an operation which is not actually executable). False positives can appear because our method is not able to exhaustively analyze the conditions of conditional and loop structures. Although our method assumes all actions inside these structures are reachable, it cannot always determine how many times the actions inside a loop will be executed. This information, as the Example 15 shows, may be required to discard some violations related with the cardinality constraints.

Our method is able to determine this in a number of cases (when our patterns syntactically match with the conditions of conditional and loop structures) but it assumes the worst case scenario when it cannot be sure (i.e. it supposes loops are executed once). However, to avoid this over-approximation, the user can directly decide this by herself (see Example 15). The type of queries for which our method requires the designer intervention are basic inequalities that 
a designer may easily solve by examining the operation. Another most costly solution to resolve this over-approximation could be extending the method with a simulation component (as [14]) to decide these situations.

Example 14 Remember the operation addMenu:

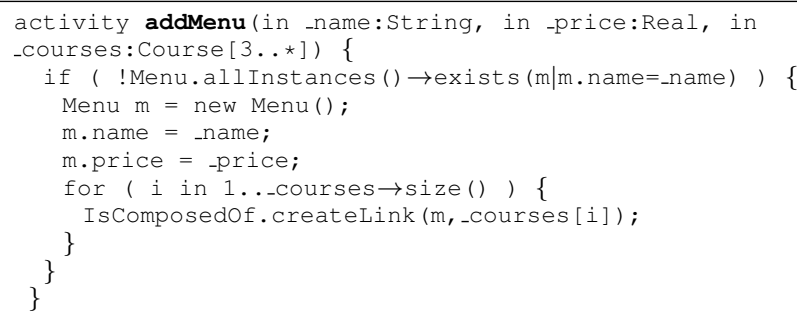

Our method is able to determine this operation is WE (since it can be successfully executed when the loop is executed at least three times). In fact, this operation is also SE since the lower multiplicity of the paramater_courses guarantees the loop will be always executed at least three times. Our method is not able to determine whether "_courses $\rightarrow$ size () $\geq 3$ " and then it assumes this inequality is not true and returns a false positive only in case of verifying if the operation is SE. However, in this case the user can intervene to resolve this fact (see Figure 21).

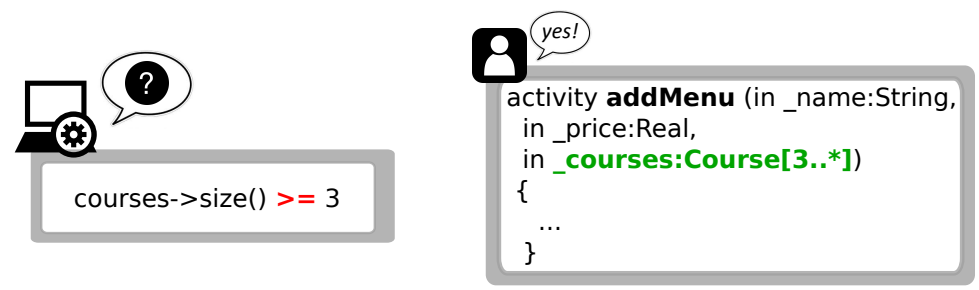

Figure 21: Example of designer intervention.

Our method covers the most commonly used integrity constraints. However, it is no suitable to address arbitrary integrity constraints, although some new constraints could be added to our patterns. In order to consider new types of integrity constraints we should proceed as follows: (1) determine the potentially violating actions (PVAs) that may violate the constraint (i.e. adding a tow to Table 3); and (2) determine the conditions to discard each detected PVA (i.e. adding a new table similar to Table 4). The effort to support new constraints will depend on the complexity of the constraints.

\section{Related Work}

A lot of research has been devoted to the problem of $\mathrm{V} \& \mathrm{~V}$ (verify and validate) UML models. In the context of UML behavioural models, there is 
a broad set of research proposals devoted to the analysis of statechart diagrams $[34,36,48]$, activity diagrams $[4,7,20,35,33,38]$, operations [13, 26, 55], xUML models $[29,60]$, or on verifying the consistent interrelationship between them $[9,27]$, among others.

We classify the closest works wrt our method in Table 6 and position it in relation with them. For each approach (1st column) we indicate the kind of behavioural model targeted (2nd column), the integrity constraints that are supported when analyzing the models (3rd column), whether UML actions can be added to specify fine-grained details of the model (4th column), the main correctness properties addressed by the method (5th column), the employed method during the verification (6th column), the type of feedback provided (7th column) and the existence of a tool publicly available to use each approach (8th column).

As Table 6 shows, half of the works do not support the definition of UML action sequences as part of the specification of their input behavioural models (even when this is indeed allowed by the UML standard). To analyze this detailed (i.e. fine-grained) specifications is precisely the focus of our method. Although there are several works addressing the verification of UML models including actions $[27,29,35,60]$, only some of them $[4,8,17,33,38]$ are aligned with the Alf action language standard.

On the other hand, works dealing with the executability of operations depart from declarative operations specified by means of pre and postconditions contracts, instead of using imperative operations. A comparison between declarative and imperative specifications is beyond the scope of this paper but it is worth to note that there are methods that transform declarative operations into imperative ones [14]. Once operations are transformed we can use the method presented herein to verify them in an lightweight way.

Most of the above works simulate the behavioural models by translating them into Model Checking [5], Constraint Programming [30] or Query Containment [21], and thus, they tend to present scalability issues. For instance, model checkers work by generating and analyzing all the potential executions at run-time and evaluating if for each (or some) execution scenario the given property is satisfied. Even with the several optimizations available (as partial order reduction or state compression), methods based on model checking techniques suffer from the state-explosion problem (i.e. the number of potential executions to analyze grows exponentially) compromising the efficiency of the method.

Regarding the type of feedback provided, some of the above methods just provide a binary response (if the model satisfies the given property or not) and, at most some provide example execution traces (counter-example) that do (not) satisfy the property. None clearly identify the source of the problems nor assist the designer to repair them. Instead, a strong point of our method is the kind of feedback, that helps the designer repairing her models.

Finally, most of the works use existing verification tools to perform the core of their analysis. However, only few works provide a tool publicly available $[13,26,38]$, even though others state they have a tool but it is not publicly 


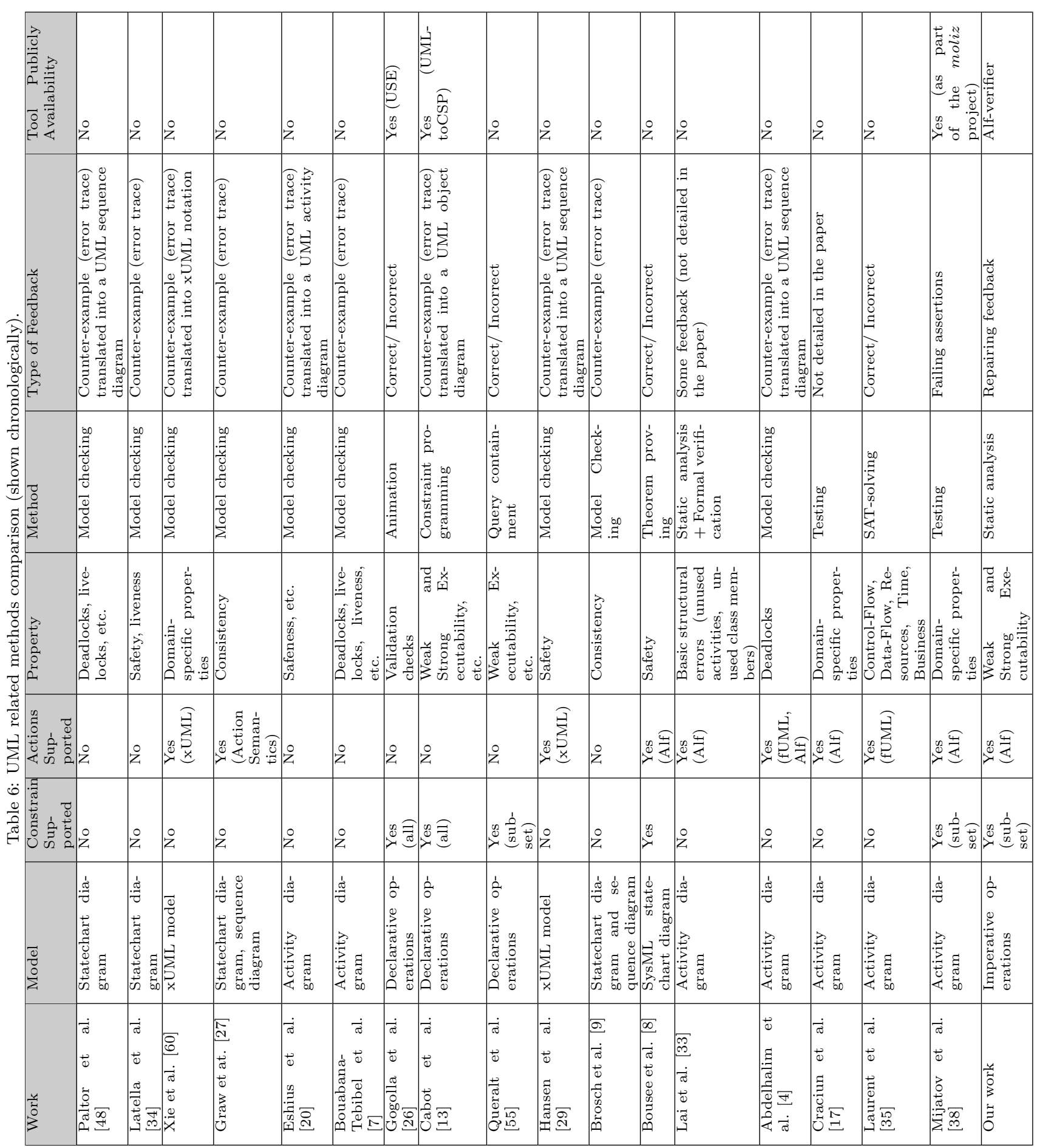


available $[4,35,48]$. This and the fact that existing approaches consider different input models, support the verification of different properties, and provide limited feedback, make it difficult to perform a fair comparison between our method and other related implementations.

To sum up, our method is the only one that deals with the verification of UML operations including actions and provides repairing feedback.

\section{Conclusions and Further Work}

We have proposed a lightweight and static method for assisting designers during the specification of executable behavioural models. In particular, our method verifies the weak and the strong executability of action-based UML operations defined by means of the new standard Alf action language wrt the structural constraints imposed by the domain model.

The main features of our method are that it is lightweight since it directly reason over a model formalized in Alf language and it is based on a static analysis of the model (no execution is required). This leads on a method that can be easily integrated in the current software development processes and CASE tools. But the more distinguishable feature comparing with other methods is that our method provides a repairing feedback to help the designers improve her models. Our method returns either a positive answer (meaning that the model achieves the checked property) or a corrective feedback (otherwise) which is expressed in the same language used to express the input model.

As a trade-off, our method supports a limited (but still useful) set of integrity constraints and it may require the designer intervention in order to return a more precise result. For these reasons, we believe the method presented in this paper could be used to perform a first correctness analysis, as a basis to ensure a fundamental quality level on action-based operations. Then, designers could proceed with a more detailed analysis adapting other methods (such as model checking) to perform a more complete verification (see Figure 22).

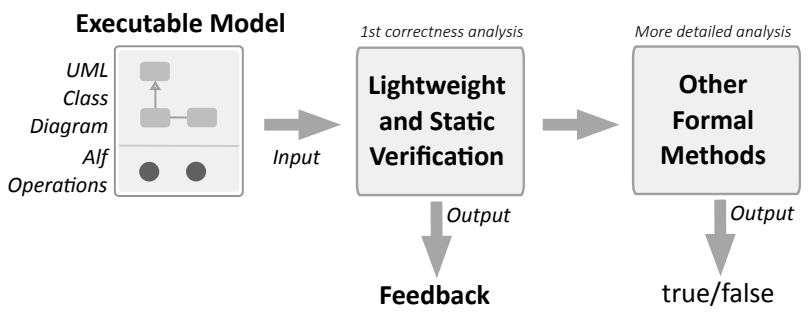

Figure 22: Connection with other verification methods.

As a further work, we plan to study the executability of operations when they are included in other UML behavioural diagrams and explore the integration of our method in a more complete verification framework that could help designers to choose the most appropriate verification technique for the model 
they have defined, depending on the target property and the verification tradeoffs (expressiveness, completeness, efficiency,...) they are ready to accept. We also plan to study how concurrent operations could be addressed in our method.

Finally, we plan to improve the usability of the prototype tool that implements our method in several ways: (1) improving the expressiveness of the addressed models to support new types of integrity constraints and to support operations defined as part of other UML diagrams such as activity diagrams; (2) improving the usability of the tool by automatically applying the feedback to fix the detected errors and by automatically removing the potentially violating actions discarded by the designer.

Acknowledgments. This work was partially supported by the Spanish funded project EOSSAC, TIN2013-44641-P.

\section{References}

[1] Alf-verifier: A lightweight tool for verifying UML-Alf executable models, https://github.com/som-research/alf-verifier.

[2] Alf-verifier: Example of use, https://goo.gl/pYEI9F.

[3] Xtext, www.xtext.org/ (Last visit September 2015).

[4] I. Abdelhalim, S. Schneider, and H. Treharne. An integrated framework for checking the behaviour of fUML models using CSP. STTT, 15(4):375-396, 2013.

[5] R. Alur. Model Checking: From Tools to Theory. In 25 Years of Model Checking, pages 89-106, 2008.

[6] J. Arsac and Y. Kodratoff. Some Techniques for Recursion Removal from Recursive Functions. ACM Trans. Program. Lang. Syst., 4(2):295-322, Apr. 1982.

[7] T. Bouabana-Tebibel and M. Belmesk. An Object-Oriented Approach to Formally Analyze the UML 2.0 Activity Partitions. Information \& Software Technology, 49(9-10):999-1016, 2007.

[8] E. Bousse, D. Mentré, B. Combemale, B. Baudry, and K. Takaya. Aligning SysML with the B Method to Provide V\&V for Systems Engineering. In Model-Driven Engineering, Verification, and Validation 2012 (MoDeVVa 2012), Innsbruck, Autriche, Sept. 2012.

[9] P. Brosch, U. Egly, S. Gabmeyer, G. Kappel, M. Seidl, H. Tompits, M. Widl, and M. Wimmer. Towards Scenario-Based Testing of UML Diagrams. In TAP, pages 149-155, 2012.

[10] D. Brown. An Introduction to Object-Oriented Analysis: Objects and UML in plain English. Wiley, 2002. 
[11] J. Cabot. Modeling Languages Portal. List of Executable UML Tools. http://modeling-languages.com/ list-of-executable-uml-tools/(LastvisitApril2016), 2011.

[12] J. Cabot, R. Clarisó, E. Guerra, and J. de Lara. Verification and validation of declarative model-to-model transformations through invariants. Journal of Systems and Software, 83(2):283-302, 2010.

[13] J. Cabot, R. Clarisó, and D. Riera. Verifying UML/OCL Operation Contracts. In IFM, volume 5423 of $L N C S$, pages 40-55, 2009.

[14] J. Cabot, R. Clarisó, and D. Riera. On the verification of UML/OCL class diagrams using constraint programming. Journal of Systems and Software, 93:1-23, 2014.

[15] C. Click and K. D. Cooper. Combining analyses, combining optimizations. ACM Trans. Program. Lang. Syst., 17(2):181-196, 1995.

[16] D. Costal, C. Gómez, A. Queralt, R. Raventós, and E. Teniente. Improving the definition of general constraints in UML. Software and System Modeling, 7(4):469-486, 2008.

[17] F. Craciun, S. Motogna, and I. Lazar. Towards Better Testing of fUML Models. In ICST, pages 485-486, 2013.

[18] D. Distante and S. R. Tilley. Conceptual modeling of web application transactions: Towards a revised and extended version of the UWA transaction design model. In 11th International Conference on Multi Media Modeling (MMM 2005), 12-14 January 2005, Melbourne, Australia, pages 439-445, 2005.

[19] Eclipse. fUML/Alf support in Papyrus. https://wiki.eclipse.org/ Papyrus/UserGuide/fUML_ALF, 2014.

[20] R. Eshuis. Symbolic Model Checking of UML Activity Diagrams. ACM Trans. Softw. Eng. Methodol., 15(1):1-38, 2006.

[21] C. Farré, E. Teniente, and T. Urpí. Checking query containment with the CQC method. Data Knowledge Engineering, 53(2):163-223, 2005.

[22] E. Gamma. Design Patterns: Elements of Reusable Object-Oriented Software $(A P C)$. Pearson Education, 1994.

[23] V. Garousi, L. C. Briand, and Y. Labiche. Control flow analysis of UML 2.0 sequence diagrams. In ECMDA-FA, pages 160-174, 2005.

[24] M. Genero, M. Piattini, and M. R. V. Chaudron. Quality of UML models. Information \&3 Software Technology, 51(12):1629-1630, 2009. 
[25] N. Gioldasis and S. Christodoulakis. UTML: unified transaction modeling language. In 3rd International Conference on Web Information Systems Engineering, WISE 2002, Singapore, December 12-14, 2002, Proceedings, pages 115-126, 2002.

[26] M. Gogolla, F. Büttner, and M. Richters. USE: A UML-based specification environment for validating UML and OCL. Sci. Comput. Program., 69(1$3): 27-34,2007$.

[27] G. Graw and P. Herrmann. Transformation and Verification of Executable UML Models. Electr. Notes Theor. Comput. Sci., 101:3-24, 2004.

[28] J. Gray and A. Reuter. Transaction Processing: Concepts and Techniques. Morgan Kaufmann Publishers Inc., San Francisco, CA, USA, 1st edition, 1992.

[29] H. H. Hansen, J. Ketema, B. Luttik, M. R. Mousavi, J. van de Pol, and O. M. dos Santos. Automated Verification of Executable UML Models. In FMCO, pages 225-250, 2010.

[30] M. Hanus. Programming with Constraints: An Introduction by Kim Marriott and Peter J. Stuckey, MIT Press, 1998. J. Funct. Program., 11(2):253$262,2001$.

[31] D. Harel. Biting the Silver Bullet - Toward a Brighter Future for System Development. IEEE Computer, 25(1):8-20, 1992.

[32] M. D. Jacyntho and D. Schwabe. Models and meta models for transactions in web applications. In Current Trends in Web Engineering - 10th International Conference on Web Engineering, ICWE 2010 Workshops, Vienna, Austria, July 2010, Revised Selected Papers, pages 37-48, 2010.

[33] Q. Lai and A. Carpenter. Defining and verifying behaviour of domain specific language with fUML. In Proceedings of the Fourth Workshop on Behaviour Modelling - Foundations and Applications, BM-FA '12, pages 1:1-1:7, New York, NY, USA, 2012. ACM.

[34] D. Latella, I. Majzik, and M. Massink. Automatic Verification of a Behavioural Subset of UML Statechart Diagrams Using the SPIN ModelChecker. Formal Asp. Comput., 11(6):637-664, 1999.

[35] Y. Laurent, R. Bendraou, S. Baarir, and M. Gervais. Formalization of fUML: An Application to Process Verification. In Advanced Information Systems Engineering - 26th International Conference, CAiSE 2014, Thessaloniki, Greece, June 16-20, 2014. Proceedings, pages 347-363, 2014.

[36] J. Lilius and I. Paltor. vUML: A Tool for Verifying UML Models. In ASE, pages 255-258, 1999. 
[37] S. J. Mellor. Executable UML Information Day - Keynote Presentation. http://www.omg.org/news/meetings/tc/agendas/va/xUML_ pdf/Mellor_Keynote.pdf (LastvisitSeptember2015), 2011.

[38] S. Mijatov, T. Mayerhofer, P. Langer, and G. Kappel. Testing functional requirements in UML activity diagrams. In Tests and Proofs - 9th International Conference, TAP 2015, Held as Part of STAF 2015, L'Aquila, Italy, July 22-24, 2015. Proceedings, pages 173-190, 2015.

[39] ModelDriven.org. Alf Reference Open Source Implementation. http: //modeldriven.github.io/Alf-Reference-Implementation/, 2014.

[40] F. Nielson, H. R. Nielson, and C. Hankin. Principles of Program Analysis. Springer-Verlag New York, Inc., Secaucus, NJ, USA, 1999.

[41] A. Nugroho and M. R. V. Chaudron. Evaluating the Impact of UML Modeling on Software Quality: An Industrial Case Study. In MoDELS, pages 181-195, 2009.

[42] A. Olivé. Conceptual Schema-Centric Development: A Grand Challenge for Information Systems Research. In CAiSE, pages 1-15, 2005.

[43] A. Olivé. Conceptual Modeling of Information Systems. Springer-Verlag New York, Inc., Secaucus, NJ, USA, 2007.

[44] OMG. UML 2.0 OCL Specification. (ptc/03-10-14). 2003.

[45] OMG. Concrete Syntax for UML Action Language (Action Language for Foundational UML), version 1.0.1, www.omg.org/spec/ALF (Last visit september 2015). 2013.

[46] OMG. Semantics Of A Foundational Subset For Executable UML Models (fUML), version 1.1, www.omg.org/spec/FUML/ (Last visit September 2015). 2013.

[47] OMG. UML 2.5 Normative. 2015.

[48] I. Paltor and J. Lilius. Formalising UML State Machines for Model Checking. In $U M L$, pages 430-445, 1999.

[49] I. Perseil. ALF formal. ISSE, 7(4):325-326, 2011.

[50] E. Planas. Lightweight and static verification of UML executable models. PhD Thesis. http://www.tdx.cat/handle/10803/116449 (Last visit September 2015), 2013.

[51] E. Planas, J. Cabot, and C. Gómez. Verifying Action Semantics Specifications in UML Behavioral Models. In CAiSE, volume 5565 of LNCS, pages 125-140. Springer, 2009. 
[52] E. Planas, J. Cabot, and C. Gómez. Lightweight Verification of Executable Models. In ER, volume 6998 of $L N C S$, pages 467-475. Springer, 2011.

[53] E. Planas, J. Cabot, and C. Gómez. Two Basic Correctness Properties for ATL Transformations: Executability and Coverage. In $M t A T L$, pages 1-9, 2011.

[54] B. Pugh and A. Loskutov. FindBugs: A Static Analyzer Tool for Java Code, http://findbugs.sourceforge.net.

[55] A. Queralt and E. Teniente. Reasoning on UML Conceptual Schemas with Operations. In CAiSE, volume 5565 of $L N C S$, pages 47-62, 2009.

[56] H. Saiedian. An Invitation to Formal Methods. Computer, 29(4):16-17, Apr. 1996.

[57] E. Seidewitz. Alf Examples. https://github.com/ ModelDriven/Alf-Reference-Implementation/tree/master/ dist (LastvisitApril2016), 2013.

[58] M. J. B. Stephen J. Mellor. Executable UML: A Foundation for ModelDriven Architecture. Addison-Wesley, 2002.

[59] UML2Tools. http://www.eclipse.org/modeling/mdt/?project=uml2tools (Last visit September 2015) .

[60] F. Xie, V. Levin, and J. C. Browne. Model Checking for an Executable Subset of UML. In ASE, pages 333-336, 2001. 